\title{
Disagreement and Learning in a Dynamic Contracting Model $^{*}$
}

\author{
Tobias Adrian \\ Mark M. Westerfield \\ Federal Reserve Bank of New York University of Southern California \\ June, 2007
}

\begin{abstract}
We present a dynamic contracting model in which the principal and agent disagree about the resolution of uncertainty, and we illustrate the contract design in an application with Bayesian learning. The disagreement creates gains from trade that the principal realizes by transferring payment to states that the agent considers relatively more likely, changing incentives. The interaction between incentive provision and learning creates an intertemporal source of "disagreement risk" that changes second-best risk sharing. There is a regime shift between economies with small and large belief differences, and an early shock to beliefs can lead to large persistent differences in variable pay even after beliefs have converged. The principal's value function is convex in the underlying belief differences because the more optimistic the agent relative to the principal, the sharper the incentives and the lower the agent's required compensation; the result is a value to uncertainty and learning. Under risk-neutrality, "selling the firm" to the agent does not implement the first-best because it precludes state-contingent trades.
\end{abstract}

JEL Classifications: D0, D8, G0

Keywords: Dynamic Contracts, Heterogeneous Beliefs, Hidden Action, Principal-Agent, Continuous Time

*Adrian can be reached at Capital Markets Research, Federal Reserve Bank of New York, 33 Liberty Street, New York, NY 10045; tobias.adrian@ny.frb.org; http://nyfedeconomists.org/adrian/. Westerfield can be reached at Department of Finance and Business Economics, University of Southern California, Hoffman Hall-701, MC-1427, 701 Exposition Blvd., Los Angeles, CA 90089-1427; mwesterf@usc.edu; http://wwwrcf.usc.edu/ ${ }^{\sim}$ mwesterf/.

We are grateful to Daron Acemoglu, Markus Brunnermeier, Douglas Gale, Ken Garbade, Bengt Holmstrom, Dirk Jenter, Oguzhan Ozbas, Stephen A. Ross, Berk Sensoy, David Sims, Costis Skiadas, Michael Steinberger, Jianfeng Yu, Jan Zabojnik, and seminar participants at Northwestern University, Princeton University, the University of Southern California, and the Federal Reserve Bank of New York for their helpful insights and comments.

The views expressed in this paper are those of the authors and do not necessarily represent those of the Federal Reserve Bank of New York or the Federal Reserve System. 


\section{Introduction}

In organizations such as financial institutions, economic agents with potentially heterogeneous beliefs interact in hierarchical and non-market-mediated environments. Those belief differences persist across both vertical and horizontal relationships, and they have been shown to affect compensation arrangements in, for example, the compensation of entrepreneurs (Landier and Thesmar (2006)) and CFOs (Ben-David, Graham, and Harvey (2006)). While there is a great deal of literature on the static effects of belief differences and on the effects of heterogeneous beliefs on asset markets ${ }^{1}$, there is very little work on the dynamic affects of differing beliefs on contracts, particularly involving learning. We present a dynamic contracting model that allows us to study the affects of disagreement and learning.

Our model consists of a principal who hires an agent to manage a project, and the two do not share beliefs about the underlying evolution of the project. The principal and agent have heterogeneous beliefs about the probability distribution of the random innovations in the economy. In this way, our model is one of heterogeneous beliefs, where the disagreement is over the project's profitability, rather than overconfidence, where one participant believes his actions are more effective than they actually are.

In this model, we are explicitly assuming that the principal and agent can agree to disagree, so that after signing the contract they still do not agree on the project's evolution. There are two main justifications for this assumption, and they amount to a rejection of two conditions for the agreement theorem of Aumann (1976). The theorem states that economic agents cannot agree to disagree if 1) they have common priors and 2) they use Bayes rule to update beliefs. The first requirement leads to undesirable economic outcomes, such as no trade (see Milgrom and Stokey (1982)); it has come under theoretical attack in Gul (1998) and models such as Acemoglu, Chernozhukov, and Yildiz (2007); and loosening it has led to interesting results (see, for example, Yildiz (2003) in the context of bargaining). The second requirement of Bayesian learning has been widely questioned in the behavioral finance and economics literature (see Mullainathan and Thaler (2001) and Baker, Ruback, and Wurgler (2006) for surveys).

\footnotetext{
${ }^{1}$ For surveys of the behavioral literature, which includes some work on heterogeneous beliefs, please see Barberis and Thaler (2003) and Baker, Ruback, and Wurgler (2006). A selection of works that model or measure the effects of heterogeneous beliefs, overconfidence, and optimism include Benabou and Tirole (2002), Bolton, Scheinkman, and Xiong (2006), Gervais, Heaton, and Odean (2006), Gervais and Goldstein (2004), Ito (1990), Jenter (2004), and Van den Steen (2001).
} 
We specify a general dynamic contracting environment that includes moral hazard (hidden action) where differences in beliefs are modeled as subjective probabilities that can be generated either by Bayesian learning with heterogeneous priors or by non-Bayesian updating. This notion of subjective probabilities dates back to at least Savage (1954) and Anscombe and Aumann (1963). Empirical work documenting the importance of heterogeneous beliefs abounds. For example, Ito (1990) finds belief heterogeneity among currency traders within and between firms. Odean (1999) demonstrates that investors with brokerage accounts tend to trade too often and interprets the finding as evidence of belief heterogeneity. Belief heterogeneity has also been shown to influence economic outcomes. Jenter (2004) demonstrates that some managers have systematically contrarian views, which in turn affect their decision making. Landier and Thesmar (2006) find that biased beliefs of entrepreneurs affect both the type of financing the entrepreneurs receive and their future profitability.

The first result from our model is that disagreement creates potential gains from trade and from risk shifting. The principal and agent would like to place side-bets on the resolution of the economy's underlying uncertainty. Because they are limited to non-market interaction, the participants are constrained to incorporate state-contingent trades in the optimal contract. In addition to the usual trade-off between insurance and incentive provision, the principal incorporates side-bets over states of the world into the contract. Moreover, when the participants learn through time, it induces a correlation between the total gains from side-bet trade and the project's outcome. This correlation is a new source of risk, and it changes the optimal degree of risk sharing between the principal and agent.

The purely dynamic correlation effect - disagreement risk - drives many of our results, and it is present even when the principal is risk neutral. Differences in opinion are correlated with both the project's outcome and the principal's certainty equivalent wealth through the learning process. When the principal is risk averse but the cost of employing the agent is constant, the principal's wealth level is correlated with both the principal's learned assessment of the project's future profitability and with the changing value of side-bets based on beliefs. When the principal is risk neutral but the cost of employing the agent varies with the agent's wealth, the cost of employment is correlated with the project's outcome which drives belief differences, the perceived future profitability of the project, and the future value of side bets. In either case, the principal's opportunities vary jointly with the portion of variable pay offered to the agent and with beliefs, and so the principal has to consider changing beliefs as an additional risk factor. This is based on correlation and is present even in states 
of the economy in which the principal and agent momentarily agree.

Disagreement risk creates a regime shift in the economy as it moves from large to moderate belief differences. When belief differences are moderate, the agent will face a contract with moderate and declining variable pay, but when belief differences are large, the agent will face large and increasing variable pay. When belief differences are moderate, the important correlation is between the project's output and the perceived future profitability of the project. This correlation is positive, and the principal responds to the increased risk by pushing more of the project onto the agent and increasing variable pay. This effect is strongest near the beginning of the economy when future profitability varies most. In contrast, when belief differences are large, the important correlation is between the project's output and the future value to side-bets. This correlation is negative, and the principal responds to the reduced risk by taking more of the project for himself, reducing variable pay. This effect is also strongest near the beginning of the economy because more of the side-bets are then in the future.

In some economies, the contractual divergence over time created by disagreement risk can be larger than the convergence of beliefs over time that occurs when the participants learn. The result is that small shocks to beliefs near the beginning of the contract can push the economy across the regime shift and result in vastly different contracts later in the economy, even if the underlying beliefs converge so as to undo the direct effects of the shock. This means that there is a fundamental instability in contract forms when the participants learn.

The gains from trade generated by differences in beliefs also create a convexity in the principal's expected payoff. Consider a principal that is pessimistic relative to the agent. When the resolution of the underlying uncertainty is low, the principal becomes more pessimistic, and he increasingly desires to "sell" consumption in high states to the agent. The principal and agent place a series of side-bets by making the agent's payment more sensitive to the underlying project. This has the additional effect of strengthening the agent's incentives in the hidden action problem. As belief heterogeneity increases, the existing trade becomes more valuable and the principal will optimally choose to increase the quantity of trade. The result is that the value the principal places on his relationship with the agent is convex in the agent's relative optimism. Jensen's inequality then tells us that changing beliefs uncertainty in learning will be valuable to the principal.

One implication of these intuitions regards the first-best outcome. In a standard risk- 
neutral principal-agent model, the first-best can be achieved by "selling the firm" to the agent who will then choose to maximize total surplus. This is no longer the case with disagreement because such a solution precludes the principal from selling consumption to the agent in extreme states. In fact, if the principal and agent are both risk-neutral, the firstbest solution does not exist because the participants would prefer an unbounded number of side-bets in addition to any other transactions. If the agent is risk-averse, then the first-best sharing rule might be steeper than in the second-best when beliefs are very different: effort is dictated in the first-best, and so there is no penalty from motivating the very high level of effort that a steep contract in the second-best would create. However, when beliefs are only moderately different, the sharing rule in the second-best can be steeper because there is a need to incentivize the agent.

An important feature of our setup is that the optimal contract allows for flexibility in commitment. This can be economically important when contract participants change their beliefs over time. The optimal contract allows for early termination by the principal and provides sufficient incentives that the agent never wants to quit, no matter how his beliefs evolve. Thus, neither side ever becomes "disappointed" with the contract, even if one realizes that the project under management is much less valuable than originally believed. This works because the principal is able to keep the agent on the edge of indifference to termination as the agent's beliefs evolve, and the principal can always offer the agent a terminal payment. We do not require the project to run to completion.

We present an illustration of our results in the context of a Bayesian model with heterogeneous priors, in which the principal learns about the underlying profitability of the project over time. Because of the convexity in the principal's problem, the principal is better off when his own priors are uncertain and when the agent is highly optimistic. This maximizes the amount of disagreement, and the principal will offer the agent a contract with high-powered incentives.

We conduct our analysis of the principal-agent model in continuous time, as it is more easily solved than a discrete time model. In our setting, dynamic contracts can be found in closed or semi-closed form and are easy to interpret. We also believe that heterogeneous beliefs naturally live in a dynamic setting because beliefs change over time. Learning is an intrinsically dynamic mechanism, and in our setup the dynamics of beliefs enter naturally into the optimal contract design. 
Our setup is closely related to the traditional principal-agent literature, especially the seminal continuous-time model of Holmstrom and Milgrom (1987) and the intermediate consumption model of Sannikov (2006). Our derivation of the optimal contract follows Westerfield (2006). Related continuous-time principal-agent models are presented in Schättler and Sung (1993) and Cvitanić and Zhang (2006). While, to our knowledge, the study of optimal contracting with heterogeneous beliefs in a dynamic principal-agent setting is new to this paper, static principal-agent models have been used to analyze situations with heterogeneous beliefs in papers such as Gervais, Heaton, and Odean (2006) and Benabou and Tirole (2002). Another example of conceptually related work is Van den Steen (2001), who studies a variety of managerial problems in the presence of differing beliefs. In addition, Bolton, Scheinkman, and Xiong (2006) study a dynamic model of CEO compensation under heterogeneous investor beliefs. Our primary contributions relative to these papers are 1) a general problem, 2) a simple and intuitive solution, and 3) flexibility of application.

Section 2 presents the basic CARA-Terminal Consumption model, while section 3 describes the optimal contract. We specialize the economy to Bayesian learning in section 4 and analyze the results. In section 5 we generalize the model to allow for intermediate consumption and more general preferences. Section 6 concludes.

\section{The Basic Model}

In this section we will lay out a simple model of the principal-agent economy. We will use as a baseline the very well understood CARA-Terminal Consumption model of Holmstrom and Milgrom (1987). On top of that will add a very general disagreement or difference in beliefs between the principal and agent. The Holmstrom and Milgrom (1987) model is ideal for our purposes because it allows us to trace the effects we find directly back to our assumptions about beliefs. Later, (section 5) we will allow for heterogenous beliefs in an economy with intermediate consumption and more general preferences.

\section{Opportunities}

There are two participants, a principal and an agent. The principal owns a project that he hires the agent to manage. The project's output is on $[0, T]$ and is driven by a standard Brownian motion $B^{\Gamma}$ on a complete probability space $(\Omega, \mathcal{B}, \Gamma)$. The project pays $Y_{T}$ at time 
$T$, where $Y_{0}$ is a constant and

$$
d Y_{t}=\mu_{t} d t+\sigma_{t} d B_{t}^{\Gamma}
$$

where $\mu_{t}$ is the agent's effort level and $\sigma_{t}$ is the project's volatility. The path of $Y$ is observable to both the principal and the agent, but the path of $B$ is observable only to the agent. $\mathcal{B}_{t}$, which is the augmented filtration generated by $B^{\Gamma}$, represents the agent's information set. $\mathcal{Y}_{t}$, which is the augmented filtration generated by $Y$, represents the principal's information set.

The agent's effort level (unobservable to the principal) is $\mu_{t} ; \mu_{t} \in \mathbb{R}$ is $\mathcal{B}_{t}$-measurable and it is restricted so that $\mu \in \mathcal{L}_{1}$. $\sigma_{t}$ is the project's volatility; $\sigma_{t} \in \mathbb{R}$ is $\mathcal{Y}_{t}$-measurable, and $\sigma \in \mathcal{L}_{2} .{ }^{2}$ In addition, $\sigma_{t}$ is assumed to depend only on $Y$ and not on $B$ or $\mu$ directly. So, the project can have stochastic volatility but that volatility does not reveal any information separately from $Y$.

The agent faces an opportunity cost for his effort which we model as a financial cost paid at $T$ :

$$
G_{T}=\int_{0}^{T} g\left(\mu_{t}\right) d t
$$

for some function $g$ for which $g(\mu) \in \mathcal{L}_{1}$. We assume that $g(0)=0, g^{\prime}(\cdot) \geq 0$, and $g^{\prime \prime}(\cdot)>0$. We also define

$$
j\left(\beta_{t}\right)=g^{\prime-1}\left(\beta_{t}\right)
$$

which is the inverse marginal cost of effort.

In return for the agent's labor, the principal offers the agent a contract that specifies a terminal payment $C_{T}$, payable at time $T$. The principal can only observe the path of $Y$,

\footnotetext{
${ }^{2}$ The spaces $\mathcal{L}_{1}$ and $\mathcal{L}_{2}$ are defined so that

$$
\begin{aligned}
& \mathcal{L}_{1}=\left\{X: \int_{0}^{T}\left|X_{t}\right| d t<\infty \quad \text { a.s. }\right\} \\
& \mathcal{L}_{2}=\left\{X: \int_{0}^{T} X_{t}^{2} d t<\infty \quad \text { a.s. }\right\}
\end{aligned}
$$
}


and so the principal can only use observations of $Y$ to offer the agent incentives to put forth effort. This captures the principal's imperfect information about the agent's controls. More rigorously, the principal is restricted to offering a contract for which $C_{T}$ is $\mathcal{Y}_{T}$-measurable.

The principal and agent have exponential utility functions over terminal consumption, with differing levels of risk aversion. Thus the principal's utility function is

$$
U\left(Y_{T}-C_{T}\right)=-\exp \left(-A\left(Y_{T}-C_{T}\right)\right)
$$

and the agent's utility function is

$$
u\left(C_{T}-G_{T}\right)=-\exp \left(-a\left(C_{T}-G_{T}\right)\right)
$$

\section{Beliefs}

In addition to the reference probability measure $\Gamma$, we also introduce two other probability measures, $\mathbb{P}$ for the principal and $\mathbb{A}$ for the agent. $\Gamma, \mathbb{P}$, and $\mathbb{A}$ are all mutually absolutely continuous (meaning they agree on zero-probability events to rule out arbitrage), with $\Gamma=\mathbb{A}$. Let $\xi_{t} \equiv(d \mathbb{A} / d \mathbb{P})_{t}$ denote the Radon-Nikodym derivative of the probability measure $\mathbb{A}$, with respect to $\mathbb{P}$. Then

$$
\begin{aligned}
d B_{t}^{\mathbb{P}} & =\delta_{t} d t+d B_{t}^{\mathbb{A}} \\
\xi_{t} & =\exp \left[-\frac{1}{2} \int_{0}^{t} \delta_{s}^{2} d s+\int_{0}^{t} \delta_{s} d B_{s}^{\mathbb{P}}\right]
\end{aligned}
$$

We assume that $\delta \in \mathcal{L}_{2}$, and $\xi_{t}$ is a martingale on $[0, T]$. We will also assume that $\delta_{0}$ and the functional form of the evolution of $\delta$ are known to all parties. We do not require any particular type of learning, so we can allow

$$
d \delta_{t}=f(t, \cdot) d t+g(t, \cdot) d Y_{t}
$$

for any $f(t, \cdot)$ and $g(t, \cdot)$ that are $\mathcal{Y}_{t}$-measurable, integrable, and fulfill the martingale requirement for $\xi_{t}$. Later, when we assume a particular type of learning in an application, we will restrict the evolution of $\delta$. However, as currently stated, $\delta$ simply describes the difference between the principal's and agent's priors over paths of the Brownian motion. 
From (1) and (4) we have

$$
\begin{aligned}
d Y_{t} & =\mu_{t} d t+\sigma_{t} d B_{t}^{\mathbb{A}} \\
d Y_{t} & =\left(\mu_{t}-\sigma_{t} \delta_{t}\right) d t+\sigma_{t} d B_{t}^{\mathbb{P}}
\end{aligned}
$$

where the first equation expresses the agent's beliefs about the project and the second expresses the principal's beliefs. It is important that we have not defined an objective probability measure, only a reference one. We take no stand on whether the principal, the agent, or both, are objectively wrong. ${ }^{3}$

We make no statement on the source of the disagreement between the principal and the agent, nor do we make any statement on the evolution of the differences in beliefs. Thus, the disagreement could be caused by heterogeneous priors, non-Bayesian learning, or a variation on "noise trading." We require only that both sides are aware of the initial magnitude of the difference and the functional form of its evolution. Similarly, $\delta_{t}$ may have any evolution, representing any type of learning process, as long as that process is known to both sides. It is not required that beliefs converge over time.

\section{The Principal's Problem}

We will use subjective welfare analysis: the agent's actions and associated objective function are taken with respect to the agent's probability measure $\mathbb{A}$, while the principal's actions and associated objective function are taken with respect to the principal's probability measure $\mathbb{P}$. This reflects the fact any learning that takes place is incorporated into the subjective probability measures and the participants cannot observe any objective probability measure.

The agent has an outside opportunity that he values with a certainty equivalent utility of $\hat{U}$. The agent will accept the principal's contract only if

$$
\max _{\mu} \mathrm{E}^{\mathbb{A}}\left[-\exp \left(-a\left(C_{T}-G_{T}\right)\right)\right] \geq \hat{U}
$$

Assuming the agent accepts the contract, his problem is to find $\mu^{*}$ so that

$$
\begin{aligned}
\mu^{*} \quad & \in \quad \arg \max _{\mu} \mathrm{E}^{\mathbb{A}}\left[-\exp \left(-a\left(C_{T}-G_{T}\right)\right)\right] \\
\text { s.t. } & d Y_{t}=\mu_{t} d t+\sigma_{t} d B_{t}^{\mathbb{A}}
\end{aligned}
$$

\footnotetext{
${ }^{3}$ In addition, because $\mathbb{P}$ and $\mathbb{A}$ are mutually absolutely continuous, their augmented filtrations, $\mathcal{B}_{t}^{\mathbb{A}}$ and $\mathcal{B}_{t}^{\mathbb{P}}$ agree, and so we will simply write $\mathcal{B}_{t}$.
} 
If $\mu^{*}$ solves the agent's problem for $C_{T}$, then we say that $C_{T}$ implements $\mu^{*}$.

The principal's problem is to maximize his objective function subject to the constraints that the agent 1) accepts the contract and 2) behaves optimally. Thus, the principal's problem is to find $C_{T}^{*}$ so that

$$
\begin{array}{lll}
C_{T}^{*} & \in \quad \arg \max _{C_{T}} \mathrm{E}^{\mathbb{P}}\left[-\exp \left(-A\left(Y_{T}-C_{T}\right)\right)\right] \\
\text { s.t. } & \text { (i) } \mu[C] \text { solves the agent's problem } \\
& \text { (ii) } d Y_{t}=\left(\mu_{t}[C]-\sigma_{t} \delta_{t}\right) d t+\sigma_{t} d B_{t}^{\mathbb{P}} \\
& \text { (iii) }\left.\mathrm{E}^{\mathbb{A}}\left[-\exp \left(-a\left(C_{T}-G_{T}\right)\right)\right]\right|_{\mu=\mu[C]} \geq \hat{U}
\end{array}
$$

\section{Equilibrium}

An equilibrium consists of a contract $C_{T}^{*}$ and an implemented level of effort $\mu^{*} . \mu^{*}$ must be adapted to the agent's information set (be $\mathcal{B}_{T}$-measurable) and solve the agent's problem (8). $C_{T}^{*}$ must be adapted to the principal's information set (be $\mathcal{Y}_{T}$-measurable) and solve the principal's problem (9).

\section{The Optimal Contract}

In this section, we will describe the optimal contract as a function of the agent's choice of effort and differences in beliefs. The result will be a flexible contract form that reduces the principal's problem (9) to a basic dynamic programming problem.

\subsection{The First-Best}

Before analyzing the second-best contracting problem, let us consider the (static) first-best in which there is no information asymmetry and the principal can simply dictate the agent's choice of $\mu_{t}$. The the principal will choose a sharing rule $C_{T}$ and an optimal effort level $\mu$ so as to maximize a weighted sum of expected utilities (the central planner's problem):

$$
C_{T}^{*}, \mu^{*} \in \arg \max _{C_{T}, \mu} \mathrm{E}^{\mathbb{P}}\left[-\exp \left(-A\left(Y_{T}-C_{T}\right)\right)\right]+\lambda \mathrm{E}^{\mathbb{A}}\left[-\exp \left(-a\left(C_{T}-G_{T}\right)\right)\right]
$$


$\lambda$ is chosen so as to meet the agent's participation constraint (7). From the definition of $\mathbb{P}$ and $\mathbb{A}$, maximizing (10) is the same as maximizing

$$
\mathrm{E}^{\mathbb{P}}\left[-\exp \left(-A\left(Y_{T}-C_{T}\right)\right)-\lambda \xi_{T} \exp \left(-a\left(C_{T}-G_{T}\right)\right)\right]
$$

which can be done state-by-state. The first order conditions are

$$
\begin{aligned}
g^{\prime}\left(\mu_{t}^{*}\right) & =1 \\
C_{T}^{*} & =\tilde{\lambda}+G_{T}^{*}+\frac{A}{A+a}\left(Y_{T}^{*}-G_{T}^{*}\right)+\frac{1}{A+a} \ln \left(\xi_{T}\right)
\end{aligned}
$$

With the exception of the term with $\xi_{t}$, both expressions above are completely standard from homogeneous beliefs models: the principal chooses the effort level that maximizes social surplus, and the agent's consumption has a constant term from the participation constraint as well as first best risk sharing of the total surplus from the project, $Y_{T}-G_{T}$.

When the principal and agent disagree, however, there is a new term that represents side-bets between the principal and agent. Because they disagree, consumption in a given state is optimally shifted towards the participant that thinks that state is more likely. When $\xi_{T}>1$, it means that the observed path of $B$ or $Y$ had a higher probability in the agents's beliefs than in the principal's beliefs, and so consumption is shifted towards the agent. The reverse holds when $\xi_{T}<1$. Intuitively, differences in beliefs create gains from trade that the principal realizes by transferring consumption based on relative beliefs.

One important implication of the first-best sharing rule is that one can never achieve the first-best by "selling the firm to the agent". This is because doing so implies that the principal and agent are not engaging in any side-bets or belief based trade and so the gains from that trade are never realized.

It is also the case that the first-bet fails to exist when both the principal and agent are risk neutral $(A=a=0)$. In this case, the optimal level of side-bets is unbounded. In fact, we can see that the level of side-bets (risk sharing based on beliefs) declines in both the risk aversion of the principal and the risk aversion of the agent, but the risk sharing payment to the agent based on project output increases in the principal's risk aversion. This is total project risk is exogenously fixed by production technology and can only be transferred, but "belief risk" is endogenous to the sharing rule and can be created and destroyed.

While the first-best formulas in this section are simple, they cannot answer two important questions: How does the sharing rule respond to shocks in a dynamic model, and how does 
the information asymmetry in the second-best affect optimal risk sharing? In other words, given the learning process, is there a difference between an innovation at time $t=T / 3$ and $t=2 T / 3$ ? We address that, along with the second-best, in the next sections.

\subsection{The Dynamic Contract}

To look at the dynamics of $C_{T}$, we need to express $C_{T}$ as a function of innovations in the project's output. We will choose to make $C_{T}$ the terminal value of the process $C_{t}$, guided by the agent's certainty equivalent wealth: $-e^{-a\left(C_{t}-G_{t}\right)}=\mathrm{E}_{t}^{\mathbb{A}}\left[-e^{-a\left(C_{T}-G_{T}\right)}\right]$. Then $C_{t}$ is an "accrual" process that can be thought of as a fund that the agent receives at time $T$ and to which the principal adds or subtracts as he observes innovations in $Y$.

Proposition 1 [Optimal Contracts]: Assume a given contract $C_{T}$ solves the principal's problem. Then the contract implements $\mu_{t}^{*}$ if and only if $C_{T}$ is the terminal value of the $C_{t}$ process with $\hat{U}=-\exp \left(-a C_{0}\right)$ and

$$
d C_{t}=g\left(\mu_{t}^{*}\right) d t+a \frac{1}{2} \beta^{2}\left(t, \mathcal{Y}_{t}\right) \sigma_{t}^{2} d t+\beta\left(t, \mathcal{Y}_{t}\right)\left(d Y_{t}-\mu_{t}^{*} d t\right)
$$

for which $\beta\left(t, \mathcal{Y}_{t}\right)$ and $\mu_{t}^{*}$ are related by

$$
\mu_{t}^{*}=j\left(\beta\left(t, \mathcal{Y}_{t}\right)\right)
$$

Furthermore,

$$
\mathrm{E}^{\mathbb{A}}\left[-\exp \left(-a\left(C_{T}-G_{T}\right)\right) \mid \mathcal{B}_{t}, \mu=\mu^{*}\right]=-\exp \left(-a\left(C_{t}^{*}-G_{t}^{*}\right)\right)
$$

When $\mu_{t}$ is drawn from a discrete or bounded set, then (13) becomes $\mu_{t}^{*}=\arg \max _{\mu_{t}} \beta\left(t, \mathcal{Y}_{t}\right) \mu_{t}-$ $g\left(\mu_{t}\right)$ which can be substituted into (12). This will change $\mu_{t}^{*}$ from an explicit to an implicit function of $\beta_{t}$.

The contract is entirely stated in terms of variables observable to the principal: the agent is reimbursed for the direct cost of effort $\left(g\left(\mu_{t}^{*}\right)\right)$ and receives an insurance payment based on the level of risk in the contract $\left(\frac{1}{2} a \beta_{t}^{2} \sigma_{t}^{2}\right)$. In addition, $\beta_{t}$ represents the principal's choice of variable pay - how much of any surprise innovation in $Y_{t}$ under $\mathbb{A}$ is paid to the agent and it implements agent's incentive compatible level of effort through (13). As is standard, the more variable pay the agent receives, the more effort the agent puts forth. 


\section{Commitment: Disappointment and Firing}

A key feature of the optimal contract of theorem 1 is that it does not rely on as much commitment as the model in section 2 specified. In particular, the general setup can accommodate early termination. As long as the principal can commit to paying the agent $C_{t}$ at the time of termination, (14) says that the agent is always indifferent to staying, quitting, or being fired. Thus, there is no concern that the principal will be unable to fire the agent if the principal's valuation of the contract becomes negative over time. In addition, there is never a motivation for the agent to quit.

This flexibility in commitment is possible because $C_{t}$ rewards the agent for costs at the moment they are incurred. Thus, potentially changing state variables (like $\sigma_{t}$ ) enter the principal's problem, but not the agent's incentive compatibility constraint: (13) treats the agent's maximization decision as a repeated static problem.

\section{The Principal's Problem}

Theorem 1 shows how to construct any optimal contract around the principal's choice of $\beta_{t}$, but only as a function of shocks $\left(d Y_{t}-\mu_{t}^{*} d t\right)$ that are a surprise to the agent. Since the principal and the agent disagree, the principal believes that part of those shocks are predictable. Fortunately, while the principal and agent disagree about the evolution of $B_{t}$, $Y_{t}$ is observable to both. Thus the evolution of the payment given in (12) is an agreed upon quantity. Since the principal assumes the agent's actions are optimal, we substitute $\mu=j\left(\beta_{t}\right)$ and $d Y_{t}=\left(\mu_{t}^{*}-\sigma_{t} \delta_{t}\right) d t+\sigma_{t} d B_{t}^{\mathbb{P}}$ into (12), which becomes

$$
d C_{t}=g\left(j\left(\beta_{t}\right)\right) d t+a \frac{1}{2} \beta_{t}^{2} \sigma_{t}^{2} d t-\beta_{t} \sigma_{t} \delta_{t} d t+\beta_{t} \sigma_{t} d B_{t}^{\mathbb{P}}
$$

The principal can "observe" $\delta$ and $B_{t}^{\mathbb{P}}$ in equilibrium because the principal solves his problem under the assumption that the agent behaves optimally (9i); so, for the purposes of the principal's problem, $\mu=j\left(\beta_{t}\right)$ and $d B_{t}^{\mathbb{P}}$ is observable from $d Y_{t}{ }^{4}$

\footnotetext{
${ }^{4} \mathrm{~A}$ further note on observability: The principal knows the agent's optimal control (as a function of $B_{t}^{\mathbb{A}}$ ) for any contract he writes, because the principal can solve the agent's problem. However, knowing the agent's optimal control allows the principal to use his observations of $Y$ to infer the path and value of $B_{t}^{\mathbb{P}}$, under the assumption - which the principal makes and the contract ensures - that the agent actually uses the optimal control. Thus, the principal can write a contract based on any quantity, including $\delta_{t}$ and $B_{t}^{\mathbb{P}}$, that depend on $d Y_{t}$ and $\mu_{t}^{*}$.

The general heterogeneous beliefs problem exhibits a separability between contracting and learning. While the learning problem influences the optimal contract, the contract choice does not change the learning process.
} 
It is important to note that the objective probability measure does not appear in (15), nor is any objective probability measure necessary to even define the principal's problem. In fact, $\delta_{t}$ appears in the contract only as the difference in beliefs between the principal and agent - the portion of $d Y_{t}-\mu_{t} d t$ that the principal believes to be predictable. This illustrates a key point: what matters is the difference in beliefs, not the belief levels. The level of beliefs fixes the level of the value functions of the principle and the agent, but their actions are determined by the difference in their beliefs.

Belief heterogeneity is reflected in the term $-\beta_{t} \sigma_{t} \delta_{t}$. This represents the difference in the principal's and agent's assessments of the payment process due to their different measures. When the agent is optimistic relative to the principal $\left(\delta_{t}>0\right)$, it means that the agent believes the underlying profitability of the project is high. Since the agent is paid a portion of that project $\left(\beta_{t} d Y_{t}\right)$, the agent believes that his payment will be high. The principal, who has a lower assessment of the project's profitability, has a correspondingly lower belief about the value the agent's final payment will take. So while the project's evolution and the agent's final payment are observable and agreeable, the principal and agent disagree about the expected value those items will have.

The principal's and agent's relative views on the value of their contracts allow a sidebet, through the contract, about the outcome of the project. When the agent is relatively optimistic $\left(\delta_{t}>0\right)$ and $\beta_{t}$ is high, the agent receives a relatively high fraction of the project and a relatively high value from optimism about the project, and the principal allocates to himself to make the agent's participation constraint bind exactly. In effect, the principal and agent are engaging in the constrained trade of consumption in different states, with the principal being able to allocate all the gains from trade to himself.

Because the amount of value created by these side-bets is endogenous - it depends on the principal's choice of $\beta_{t}$ - the principal will have an incentive to alter the contract to increase the value of these bets. In fact, viewing the contract (15), one can see that as the agent becomes more optimistic ( $\delta_{t}$ increases), the convexity of $g$ and $\frac{1}{2} a \beta_{t}^{2} \sigma_{t}^{2}$ implies that the marginal cost of implementing a particular choice of $\mu^{*}$ declines. This leads us to the principal's relaxed problem:

In finding the optimal contract, we can treat the evolution of the difference in beliefs as exogenous. This makes economic sense because the contract affects only the agent's effort level, not outside business conditions or whatever else might drive the Brownian innovation term. 
Proposition 2 [The Principal's Problem]: A contract $C_{T}$ is a solution to the principal's problem (9) if and only if

$$
\begin{array}{ll}
\beta^{*} \quad \in \quad \arg \max _{\beta} \mathrm{E}^{\mathbb{P}}\left[-\exp \left(-A\left(Y_{T}-C_{T}\right)\right)\right] \\
\text { s.t. } & \text { (i) } d Y_{t}=\left(j\left(\beta_{t}\right)-\sigma_{t} \delta_{t}\right) d t+\sigma_{t} d B_{t}^{\mathbb{P}} \\
& \text { (ii) } d C_{t}=g\left(j\left(\beta_{t}\right)\right) d t+a \frac{1}{2} \beta_{t}^{2} \sigma_{t}^{2} d t-\beta_{t} \sigma_{t} \delta_{t} d t+\beta_{t} \sigma_{t} d B_{t}^{\mathbb{P}}
\end{array}
$$

where $Y_{0}=0$ and $C_{0}=-\frac{1}{a} \ln (-\hat{U})$.

This is a standard optimal control problem with one choice variable: $\beta_{t}$. As such, it can be solved analytically in numerous well-understood ways, and numerical techniques are well developed. We solved one version of it - the case of Bayesian learning from heterogeneous priors - in the next section.

\section{Bayesian Learning}

In this section, we look at the model of sections 2 and 3 under specific assumptions. We will assume that learning is Bayesian, costs and quadratic, and volatility is constant.

\subsection{The Principal's Problem}

We assume that the difference in beliefs about the growth rate of the project stems from a difference in priors that is not resolved by signing the contract. We assume that the agent does not update his beliefs, so one can view the agent as an expert who fully understands the economy, or as a noise trader who does not learn. We assume that the principal has a Gaussian prior at time 0 with prior mean $\delta_{0}$ and uncertainty $\gamma_{0}$. In addition, we assume that the agent faces a quadratic financial cost of effort ${ }^{5}, g\left(\mu_{t}\right)=\frac{1}{2} \mu_{t}^{2}$ and that volatility is constant $\left(\sigma_{t}=\sigma\right)$. The agent's incentive compatibility constraint (13) implies that $\mu_{t}^{*}=\beta_{t}$.

\footnotetext{
${ }^{5}$ In order to keep the example simple, we follow Holmstrom and Milgrom (1987) and make several simplifying assumptions such as CARA utility, constant volatility $\sigma$, and quadratic cost of effort. One additional simplification is that the cost function extends over the entire real line. We can justify this in two ways. First, the CARA utility can be viewed as an approximation to a particular section of the agent's utility function, and the cost functional as an approximation to the true cost function for a region in which the agent is not too pessimistic. Similar justifications are given in the affine term structure literature or the CARA-normal asymmetric information asset-pricing literature. A second justification is that the principal
} 
The principal uses Bayesian updating, so the prior means and uncertainties evolve as

$$
\begin{aligned}
d \delta_{t} & =-\frac{\gamma_{t}}{\sigma^{2}}\left(d Y_{t}-\mu_{t}^{*}+\sigma \delta_{t}\right)=-\frac{\gamma_{t}}{\sigma} d B_{t}^{\mathbb{P}} \\
d \gamma_{t} & =-\frac{\gamma_{t}^{2}}{\sigma^{2}} d t
\end{aligned}
$$

according to the Kalman-Bucy filter, as presented in Liptser and Shiryaev (2000).

To solve the principal's relaxed problem (16), we will use dynamic programming. To simplify the problem, we will use the state variable $W_{t}=Y_{t}-C_{t}$. After substituting in $\mu_{t}^{*}=\beta_{t}$, we have

$$
d W_{t}=\left[\beta_{t}-\sigma \delta_{t}-\frac{1}{2} \beta_{t}^{2}+\beta_{t} \sigma \delta_{t}-a \frac{1}{2} \beta_{t}^{2} \sigma^{2}\right] d t+\left(1-\beta_{t}\right) \sigma d B_{t}^{\mathbb{P}}
$$

The principal's value function, which we later verify, has the form

$$
V\left(t, W_{t}, \delta_{t}\right)=-\exp \left(-A\left(W_{t}+F(t)+G(t) \delta_{t}+H(t) \delta_{t}^{2}\right)\right)
$$

with boundary condition $V\left(T, W_{T}, \delta_{T}\right)=-\exp \left(-A W_{T}\right)$.

The Hamilton-Jacobi-Bellman equation is

$$
\begin{aligned}
0=\max _{\beta_{t}} & -A V\left(t, W_{t}, \delta_{t}\right)\left[\beta_{t}-\sigma \delta_{t}-\frac{1}{2} \beta_{t}^{2}+\beta_{t} \sigma \delta_{t}-a \frac{1}{2} \beta_{t}^{2} \sigma^{2}-\frac{1}{2} A\left(1-\beta_{t}\right)^{2} \sigma^{2}\right. \\
& +F^{\prime}(t)+G^{\prime}(t) \delta_{t}+H^{\prime}(t) \delta_{t}^{2}+A\left(1-\beta_{t}\right)\left(G(t)+H(t) 2 \delta_{t}\right) \gamma_{t} \\
& \left.+\left(H(t)-\frac{1}{2} A\left(G(t)+2 H(t) \delta_{t}\right)^{2}\right)\left(\frac{\gamma_{t}}{\sigma}\right)^{2}\right]
\end{aligned}
$$

and so the principal's optimal choice of variable pay is

$$
\beta_{t}^{*}=\frac{1+A \sigma^{2}}{1+a \sigma^{2}+A \sigma^{2}}+\frac{\sigma \delta_{t}-A \gamma_{t}\left(G(t)+H(t) 2 \delta_{t}\right)}{1+a \sigma^{2}+A \sigma^{2}}
$$

monitors the agent and makes it costly for the agent to sabotage the project. Under this interpretation, the agent pays a cost of effort when $\mu_{t}>0$ and a cost to avoid sabotage monitoring when $\mu_{t}<0$. Then, assuming no monitoring (free sabotage) is equivalent to imposing the constraint that $\beta_{t} \geq 0$. It is still possible to solve for the principal's value function with this constraint, but it makes the Hamilton-JacobiBellman equation significantly more complicated. The additional constraint does not change the nature of the solution qualitatively. 
Plugging $\beta_{t}^{*}$ in the HJB equation yields the following set of ODEs:

$$
\begin{aligned}
H^{\prime}(t) & =2 \frac{\gamma(t)}{\sigma} \frac{A \sigma^{2} H(t)}{1+a \sigma^{2}+A \sigma^{2}}+2 \frac{\gamma(t)^{2}}{\sigma^{2}} A \frac{\left(1+a \sigma^{2}\right) H(t)^{2}}{1+a \sigma^{2}+A \sigma^{2}}-\frac{\sigma^{2}}{2\left(1+a \sigma^{2}+A \sigma^{2}\right)} \\
G^{\prime}(t) & =2 A \frac{\gamma(t)^{2}}{\sigma^{2}} \frac{1+a \sigma^{2}}{1+a \sigma^{2}+A \sigma^{2}} G(t) H(t)+\sigma \frac{a \sigma^{2}-2 \sigma A a \gamma(t) H(t)+A \gamma(t) G(t)}{1+a \sigma^{2}+A \sigma^{2}} \\
F^{\prime}(t) & =-H(t) \frac{\gamma(t)^{2}}{\sigma^{2}}+\frac{-2 A G(t) \gamma(t) a \sigma^{4}-G(t)^{2} A \gamma(t)^{2}\left(1+a \sigma^{2}\right)}{2\left(1+a \sigma^{2}+A \sigma^{2}\right)}-\frac{1+A \sigma^{2}-A a \sigma^{4}}{2\left(1+a \sigma^{2}+A \sigma^{2}\right)}
\end{aligned}
$$

where $\gamma(t)=\frac{\gamma_{0} \sigma^{2}}{\gamma_{0} t+\sigma^{2}}$. The ODEs can be easily solved numerically with the boundary condition $F(T)=G(T)=H(T)=0$ (and they can be solved analytically when the principal is riskneutral: $A=0$ ). We interpret the solution to the principal's optimal control choice and welfare function in the next section.

\subsection{The Solution}

\section{Side-bets, Disagreement Risk and Effort}

The key finding is that both dynamic and static differences in beliefs determine the incentive contract. Relative to the Holmstrom and Milgrom (1987) baseline (no differences of opinion), our expression for the slope of the contract (18) and the optimal level of effort $\left(\mu_{t}^{*}\right)$ contains two extra terms:

$$
\sigma \delta_{t}-A \gamma_{t}\left(G(t)+H(t) 2 \delta_{t}\right)
$$

The first term, $\sigma \delta_{t}$, is a direct effect: when the agent is relatively more optimistic $\left(\delta_{t}>0\right)$, the principal grants steeper incentives so as to maximize the value of the contract and the side-bets on the project's outcome. When the agent is relatively pessimistic, $\beta_{t}^{*}$ declines, again because the slope of the contract creates both incentives and side-bets.

The second term, $-A \gamma_{t}\left(G(t)+H(t) 2 \delta_{t}\right)$, is a correlation effect - disagreement risk. Differences of opinion $\left(\delta_{t}\right)$ are correlated with the project's outcome $\left(Y_{t}\right)$ and with the principal's certainty equivalent value from the remainder of the contract. This correlation creates a desire to shift risk between the participants, and this can only be accomplished through the contract and hence through $\beta$. In doing so, the principal changes the effort level that the contract implements. 
Notice that the correlation effect occurs whether or not the principal and agent actually disagree at any particular time. If $\delta_{t}=0$ for some $t$, the direct effect vanishes, but the correlation effect does not because it is based on future changes. For the same reason, the correlation effect is weaker as one moves closer to the completion of the contract. The correlation effect is illustrated in figures 1 and 2 .

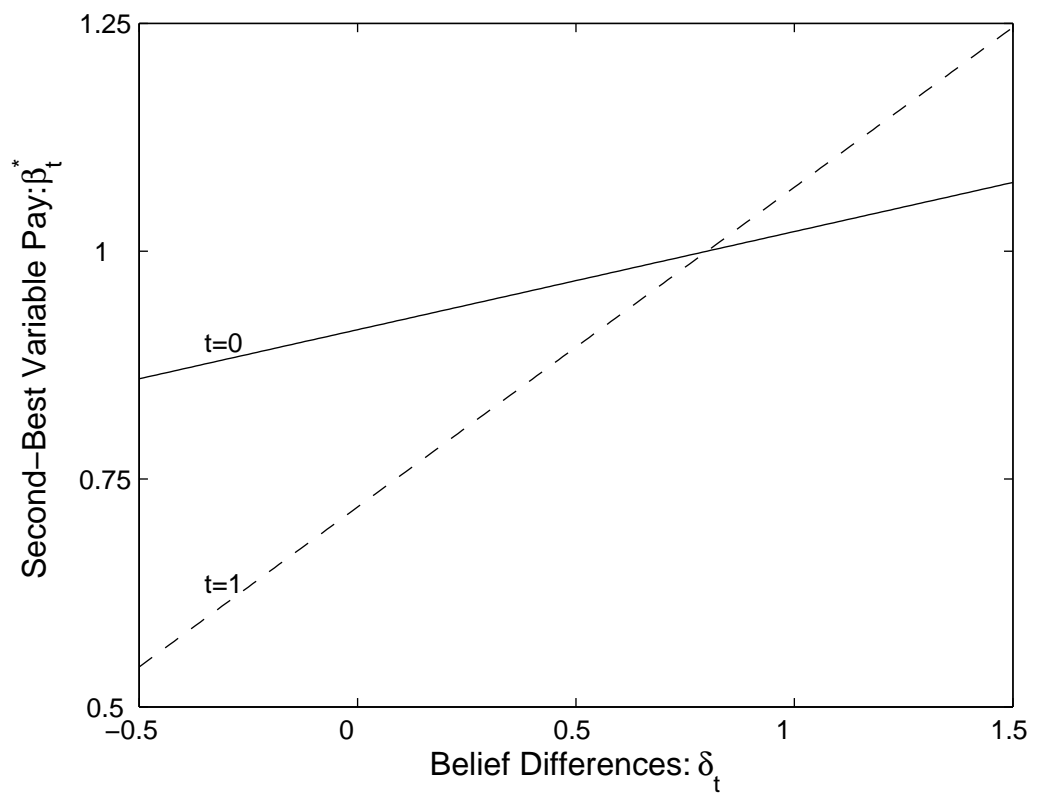

Figure 1: $\beta_{t}^{*}$ as a function of $\delta_{t}$ for $t=0$ and $t=1$. We set $A=a=T=1, \sigma=.8$, and $\gamma_{0}=5$.

The key fact illustrated in figures 1 and 2 is that there is a regime shift when ones moves from moderate belief differences to large ones. When belief differences are moderate $\left(\delta_{t}\right.$ is small), the agent's variable pay is small and decreases over time. When belief differences are large, the agent's variable pay is large and increases over time.

The regime shift happens because the principal's certainty equivalent wealth is made up of two parts: one from the value of the project itself and one from the value of the side-bets in the contract. When $\delta_{t}$ is small, project profitability dominates, the correlation between beliefs and certainty equivalent wealth is positive $(G(t)<0)$, and the principal pushes more of the project onto the agent. When $\delta_{t}$ is large, the contract dominates, the correlation is negative $(H(t)>0)$, and the principal keeps more of the project for himself. Both effects decline over time, as future correlations becomes less important as the contract 


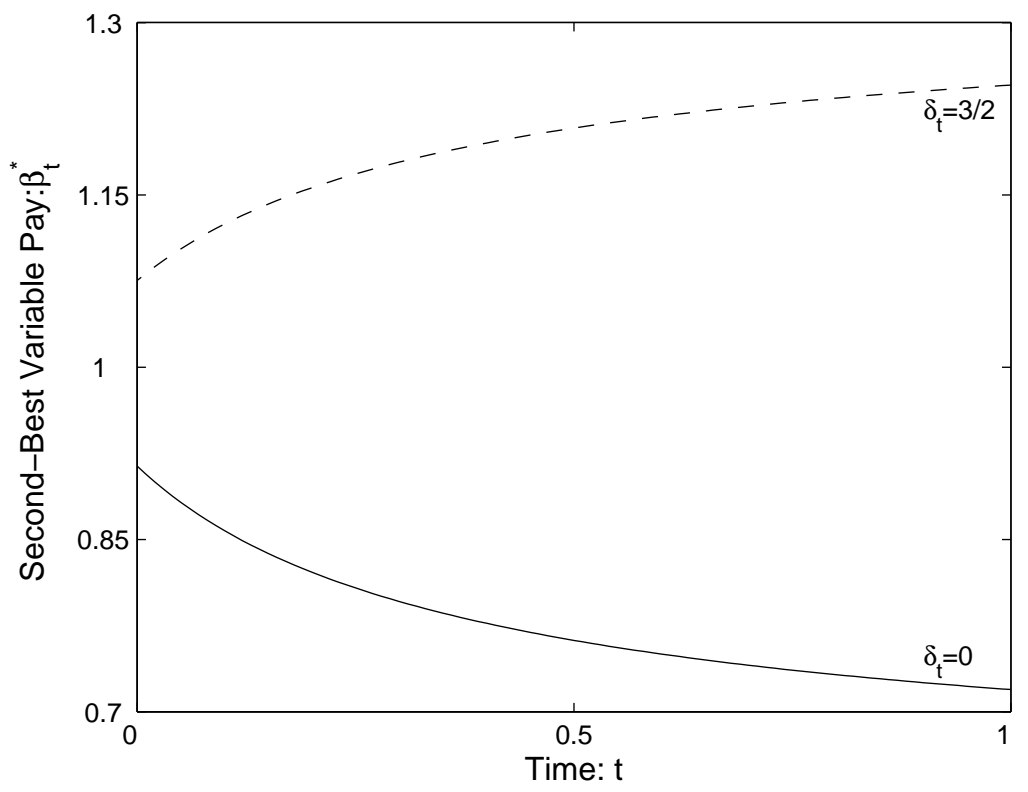

Figure 2: $\beta_{t}^{*}$ as a function of time for $\delta_{t}=0$ and $\delta_{t}=1 / 5$. We set $A=a=T=1, \sigma=.8$ and $\gamma_{0}=5$. For reference, the value of $\beta_{t}^{*}$ under homogeneous beliefs for these parameters is 0.72 .

nears completion.

\section{Disagreement Risk and Non-Convergence}

Under our model of Bayesian learning, beliefs will converge over time, but the regimes shift described as disagreement risk can cause the contract to fail to converge. The rational is illustrated in figure 2: in the cross section of economies, large belief differences lead to large and increasing variable pay, while small belief differences lead to small and declining variable pay. In some economies, this divergence in variable pay as a function of beliefs and time can actually dominate the convergence in beliefs that is simultaneously taking place. The result is illustrated in figures 3,4 and 5. These plots are impulse response function: they plot the path of the expected value of $\beta_{t}$ or $\delta_{t}$ given a shock to $\delta_{0}$ under the agent's measure $\mathbb{A}$ and the principal's measure $\mathbb{P}$.

In figure 3, beliefs converge under the agent's measure because the agent believes the principal will 'learn to agree". Under the principal's measure. there is no such convergence because the principal knows that the agent is not updating his beliefs. 

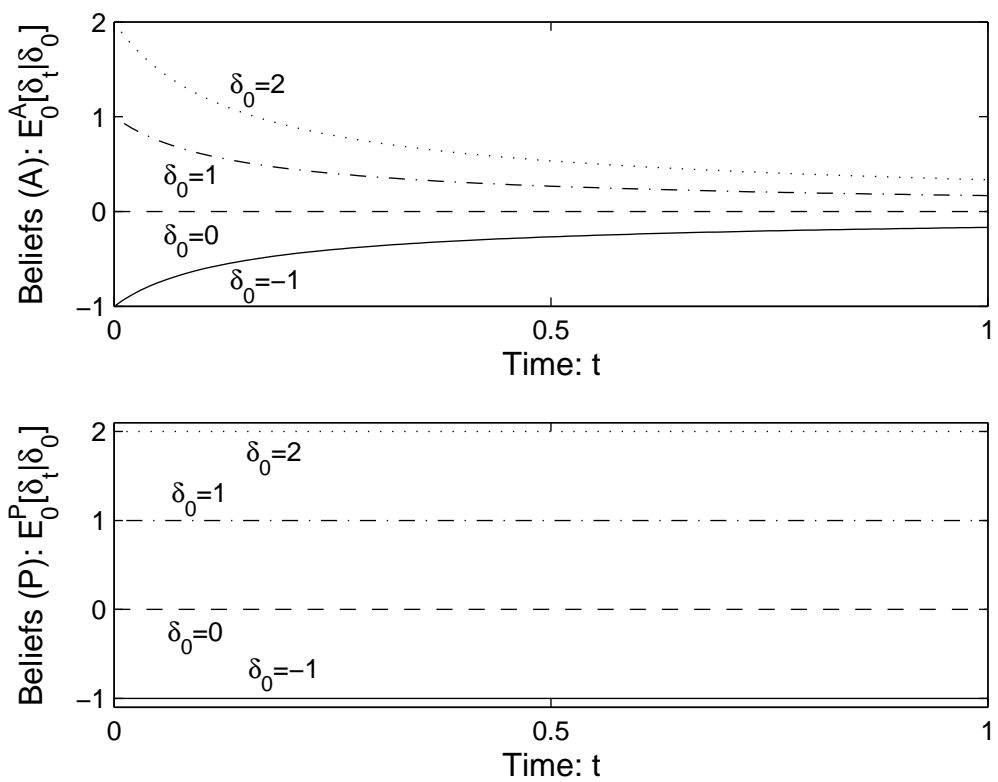

Figure 3: An impulse response function for belief differences given a shock at time 0 to beliefs. We plot $\mathrm{E}^{\mathbb{A}}\left[\delta_{t} \mid \delta_{0}\right]$ for $\delta_{0}=-1,0,1,2$. We set $A=T=1, a=.1, \sigma=.8$ and $\gamma_{0}=5$.

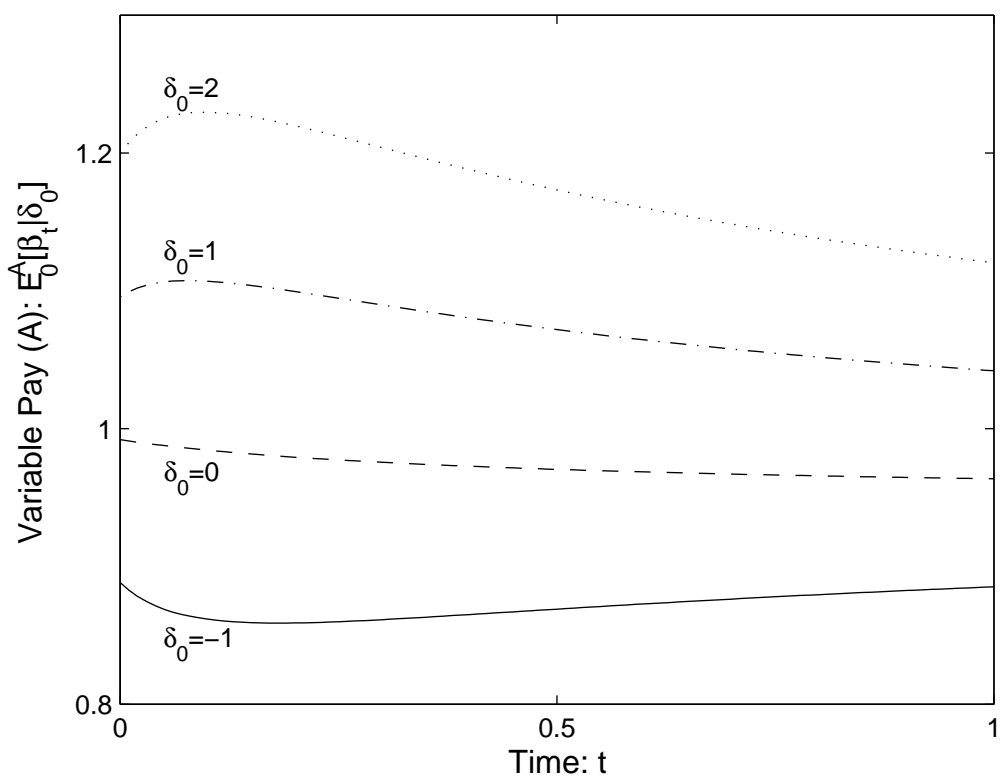

Figure 4: An impulse response function to variable pay $\left(\beta_{t}\right)$ given a shock at time 0 to beliefs. We plot $\mathrm{E}^{\mathbb{A}}\left[\beta_{t} \mid \delta_{0}\right]$ for $\delta_{0}=-1,0,1,2$. We set $A=T=1, a=.1, \sigma=.8$ and $\gamma_{0}=5$. 


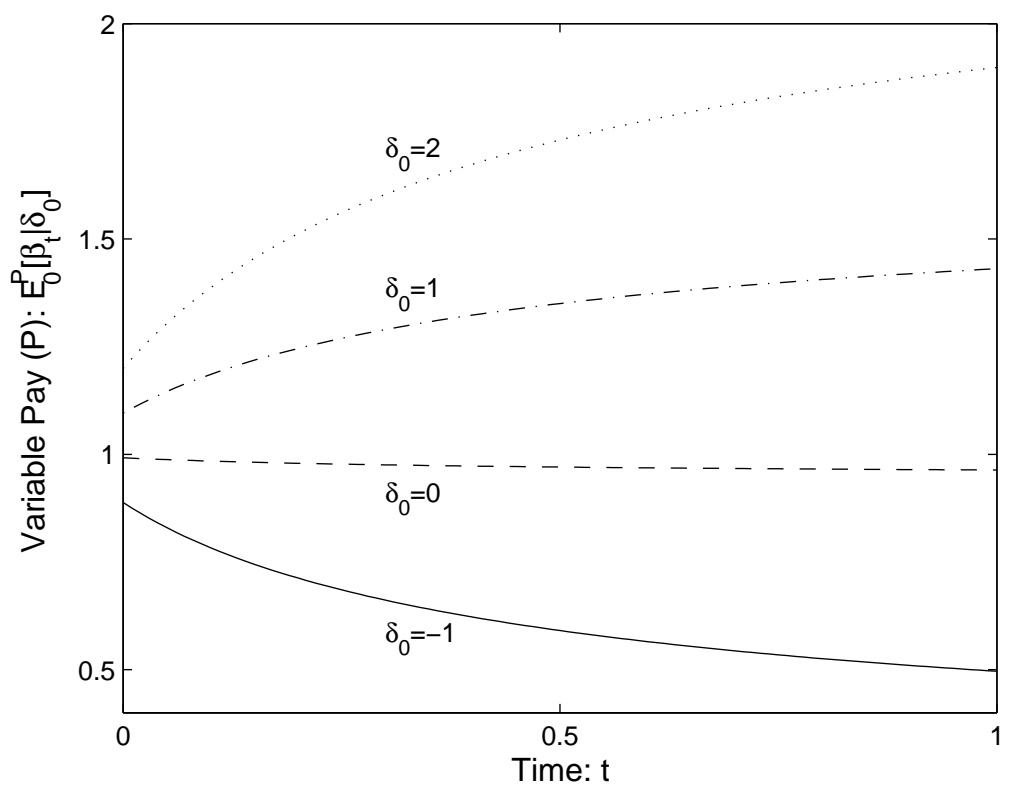

Figure 5: An impulse response function to variable pay $\left(\beta_{t}\right)$ given a shock at time 0 to beliefs. We plot $\mathrm{E}^{\mathbb{P}}\left[\delta_{t} \mid \delta_{0}\right]$ for $\delta_{0}=-1,0,1,2$. We set $A=T=1, a=.1, \sigma=.8$ and $\gamma_{0}=5$.

In figures 4 and 5 we see the effects on the contract from a shock to $\delta_{0}$. Under the agent's measure, if we held time constant and allowed beliefs to converge, the level of variable pay would converge as well. However, a large enough positive shock pushes the economy across the regime shift from disagreement risk that we saw illustrated earlier: if we held beliefs constant and vary time directly, the level of variable pay would diverge. Putting these two effects together gives the short term divergence and long term persistence pattern that we see under the agent's measure. Under the principal's measure, beliefs do not converge in expectation, and so we see only the effect of contractual divergence over time.

These plots indicate that in some simple and apparently well understood contracting models, shocks early in the economy can cause contracts to be radically different even very late in the economy. This is a striking result because it indicates that the pattern of beliefs can have large impacts on contractual relationships even after those belief differences have declined.

One might think that the non-convergence result would be diminished by taking $T$ to be very large, but this is not the case. It is true that when $T$ is very large and $t$ is near 
$T$ that we see some convergence. However when $t$ is small, the non-convergence is stronger and the overall pattern is simply a much larger version of figures 4 and 5 . This result can be understood from its source: disagreement risk exists because the principal's certainty equivalent wealth is made up of a part from the project's future profitability and a part from the future value of the contract, and those both co-vary with the principal's existing wealth. When the economy becomes longer, the magnitude of the principal's certainty equivalent wealth and its co-variances become larger. Thus, disagreement risk is more extreme and the dispersion in $\beta_{t}$ is larger as well.

\section{Convexity and Uncertainty}

We also examine the principal's certainty equivalent wealth from the differences in beliefs and the contracting process:

$$
-\frac{1}{A} \ln \left(-V\left(0, W_{0}=0, \delta_{0}\right)\right)+\sigma \delta_{0} T
$$

The latter term is added to cancel out the expected profitability of the project under the principal's measure. We wish to examine gains from contracting under heterogeneity, rather than the expected payoff from the project itself. This quantity is plotted in figure 6 .

We observe two facts in figure 6 . The first observation is that the principal's value function is convex in $\delta_{0}$. This results from combining income and substitution effects. The principal's direct cash flow from belief differences is $\beta_{t} \sigma \delta_{t}$. Therefore, as $\delta_{t}$ increases, the principal's direct cash flows increase as well. However, as $\delta_{t}$ increases, (18) and $H(t)>0$ show us that the principal increases the steepness of the agent's incentives. The first is an income effect, the second a substitution effect, and the sum is a more than linear increase in the principal's utility as a function of $\delta_{0}$.

The convexity of the value function drives the second observation: The principal has a higher expected value to the contract when $\gamma_{0}$ is large. This is because a series of surprise innovations in $B_{t}$ will cause $\delta_{t}$ to vary as if by adding a mean-preserving spread under $\mathbb{P}$ (17a). Because the principal's value function is convex in $\delta_{0}$, he is better off with relative priors that can easily change. 


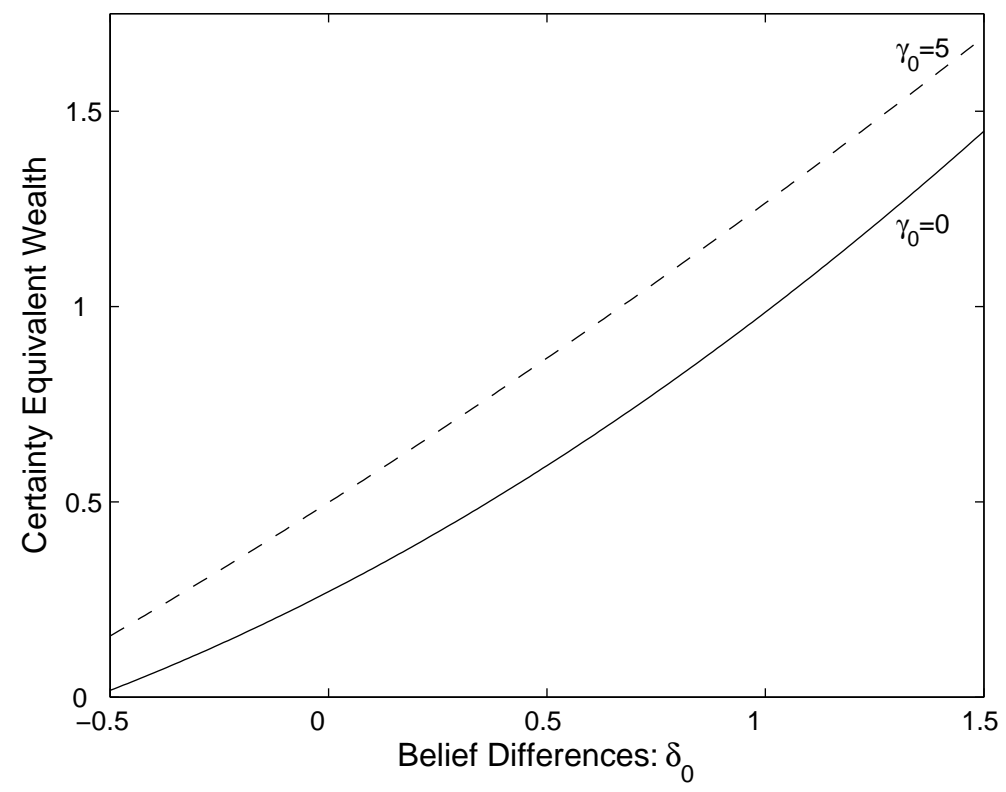

Figure 6: The principal's value to contracting is the function $-\frac{1}{A} \ln \left(-V\left(0, W_{0}=0, \delta_{0}\right)\right)+\sigma \delta_{0} T$. We plot this as a function of $\delta_{0}$ for two values of $\gamma_{0}$. The plot sets $T=a=1, \sigma=.8$ and $A=.1$. 


\subsection{Comparison to the First-Best}

The first-best contract actually does have many of the interesting features of the secondbest - disagreement risk and non-convergence - although we require a dynamic analysis to bring them out. Simply observing the apparently static first-beset (11) is not useful. Instead, we analyze $C_{t}$ as in the case of the second-best, but without imposing the incentive comparability constraint. Then the principal's first-best dynamic problem is to solve (9) without constraint (9i).

Unlike in the second-best, the principal can control $\mu_{t}$ and $\beta_{t}$ separately and so wealth evolves as

$$
d W_{t}=d Y_{t}-d C_{t}=\left[\mu_{t}-\sigma \delta_{t}-\frac{1}{2} \mu_{t}^{2}+\beta_{t} \sigma \delta_{t}-a \frac{1}{2} \beta_{t}^{2} \sigma^{2}\right] d t+\left(1-\beta_{t}\right) \sigma d B_{t}^{\mathbb{P}}
$$

and the value function will have the form

$$
\hat{V}\left(t, W_{t}, \delta_{t}\right)=-\exp \left(-A\left(W_{t}+\hat{F}(t)+\hat{G}(t) \delta_{t}+\hat{H}(t) \delta_{t}^{2}\right)\right)
$$

with boundary condition $\hat{V}\left(T, W_{T}, \delta_{T}\right)=-\exp \left(-A W_{T}\right)$. The Hamilton-Jacobi-Bellman equation is

$$
\begin{aligned}
0=\max _{\beta_{t}, \mu_{t}} & -A \hat{V}\left(t, W_{t}, \delta_{t}\right)\left[\mu_{t}-\sigma \delta_{t}-\frac{1}{2} \mu_{t}^{2}+\beta_{t} \sigma \delta_{t}-a \frac{1}{2} \beta_{t}^{2} \sigma^{2}-\frac{1}{2} A\left(1-\beta_{t}\right)^{2} \sigma^{2}\right. \\
& +\hat{F}^{\prime}(t)+G^{\prime}(t) \delta_{t}+\hat{H}^{\prime}(t) \delta_{t}^{2}+A\left(1-\beta_{t}\right)\left(\hat{G}(t)+\hat{H}(t) 2 \delta_{t}\right) \gamma_{t} \\
& \left.+\left(\hat{H}(t)-\frac{1}{2} A\left(\hat{G}(t)+2 \hat{H}(t) \delta_{t}\right)^{2}\right)\left(\frac{\gamma_{t}}{\sigma}\right)^{2}\right]
\end{aligned}
$$

and so the principal chooses

$$
\begin{aligned}
\mu_{t}^{*} & =1 \\
\beta_{t}^{*} & =\frac{A}{a+A}+\frac{\sigma \delta_{t}-A \gamma_{t}\left(\hat{G}(t)+\hat{H}(t) 2 \delta_{t}\right)}{a \sigma^{2}+A \sigma^{2}}
\end{aligned}
$$

The functions $\hat{F}(t), \hat{G}(t)$, and $\hat{H}(t)$ form a solvable system of ODEs with boundary condition $\hat{F}(T)=\hat{G}(T)=\hat{H}(T)=0$, which verifies our claim about the form of the value function.

The difference between the first- and second-best can be seen from comparing the two optimal policies, (18) and (19). The first-best also has the direct and indirect effects of 
heterogeneous beliefs through the term $\sigma \delta_{t}-A \gamma_{t}\left(\hat{G}(t)+\hat{H}(t) 2 \delta_{t}\right)$, and the effects of disagreement risk and the various correlations will run in the same direction. However, because the principal no longer has to choose $\mu_{t}^{*}$ and $\beta_{t}^{*}$ in a incentive compatible way, the principal is free to off the agent either much more or much less variable pay than would be required to motivate a desired level of effort. This is reflected in figure 7, which shows how disagreement risk changes the level of variable pay over time.

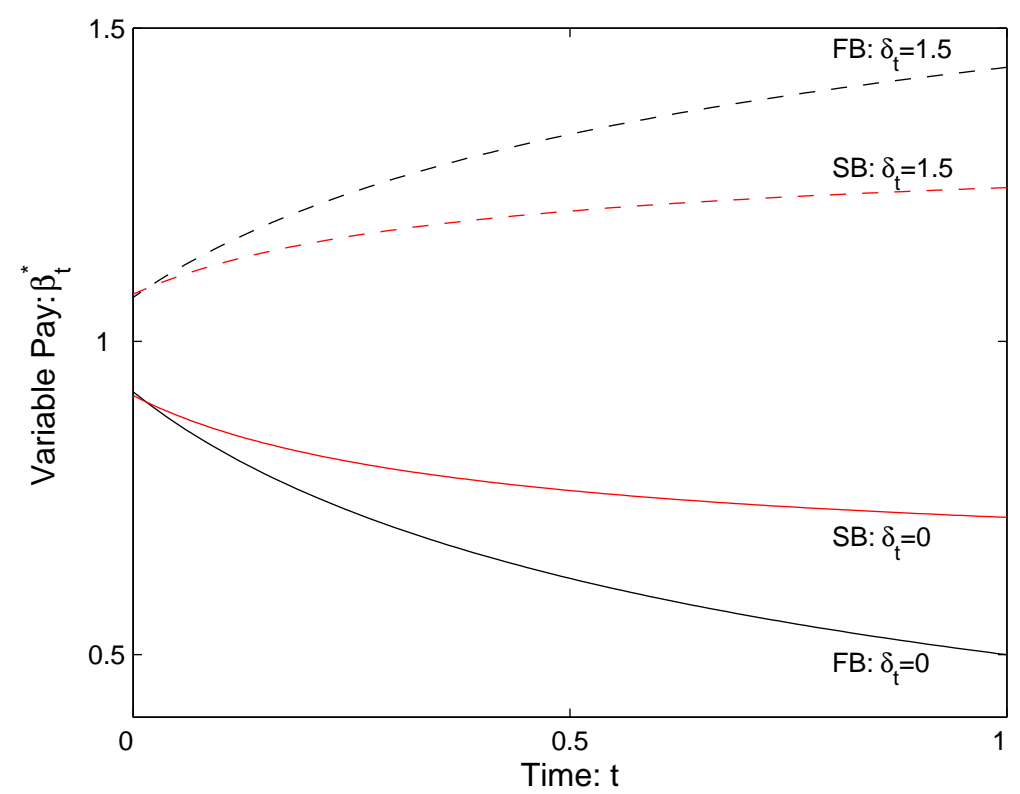

Figure 7: $\beta_{t}^{*}$ as a function of time for $\delta_{t}=0$ and $\delta_{t}=1 / 5$ in the first and second best. We set $A=a=T=1, \sigma=.8$ and $\gamma_{0}=5$. For references, the first-best value of $\beta_{t}^{*}$ under these parameter with homogeneous beliefs is 0.5 , while the second-best value is 0.72 .

The key fact in figure 7 is that the second-best contract is more moderate than the firstbest. In the first-best, the level of variable pay diverges fast over time and the regime shift between small and large belief differences is more intensive. This happens because when belief differences are large in the second-best, the principal would like undertake a very large number of side-bets but implement only a moderate level of effort. Simultaneously, when belief differences are small, the principal does not want to engage in side-bets, but must still implement effort. When effort is de-coupled from side-bets in the first-best, the level of variable pay becomes more extreme.

The de-coupling of side-bets and incentives in the first-best makes the principal's value 


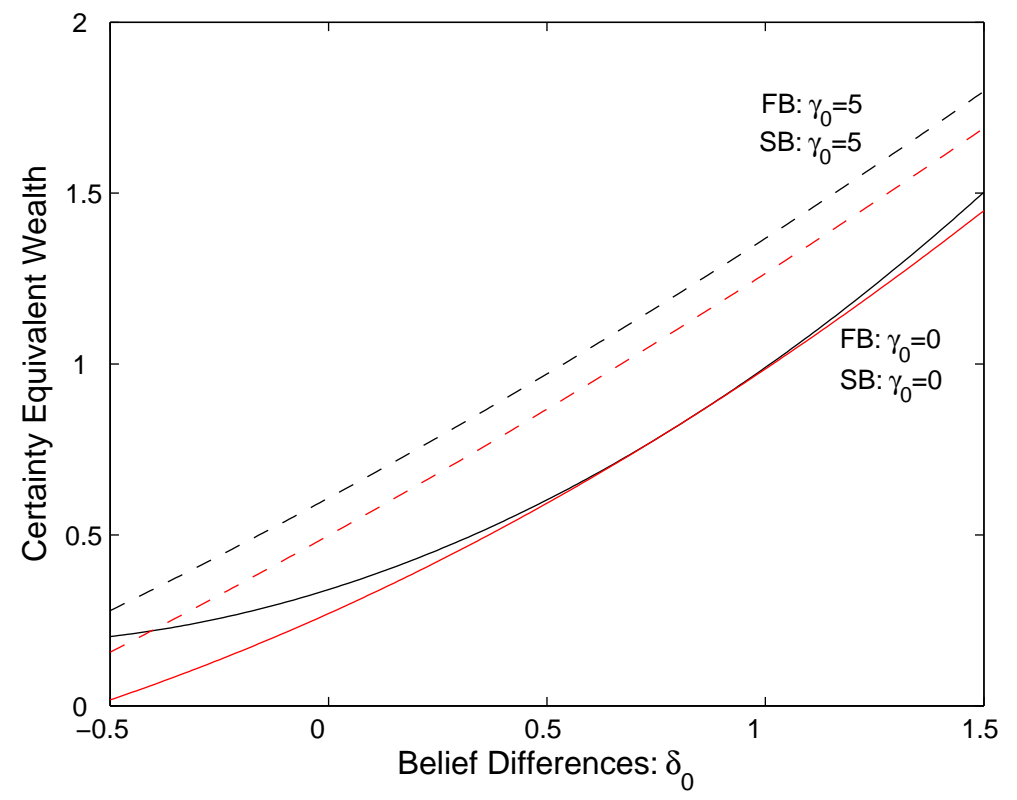

Figure 8: The principal's value to contracting is the function $-\frac{1}{A} \ln \left(-V\left(0, W_{0}=0, \delta_{0}\right)\right)+\sigma \delta_{0} T$. We plot this as a function of $\delta_{0}$ for two values of $\gamma_{0}$ for both the first- and second-best. The plot sets $T=a=1, \sigma=.8$ and $A=.1$. 

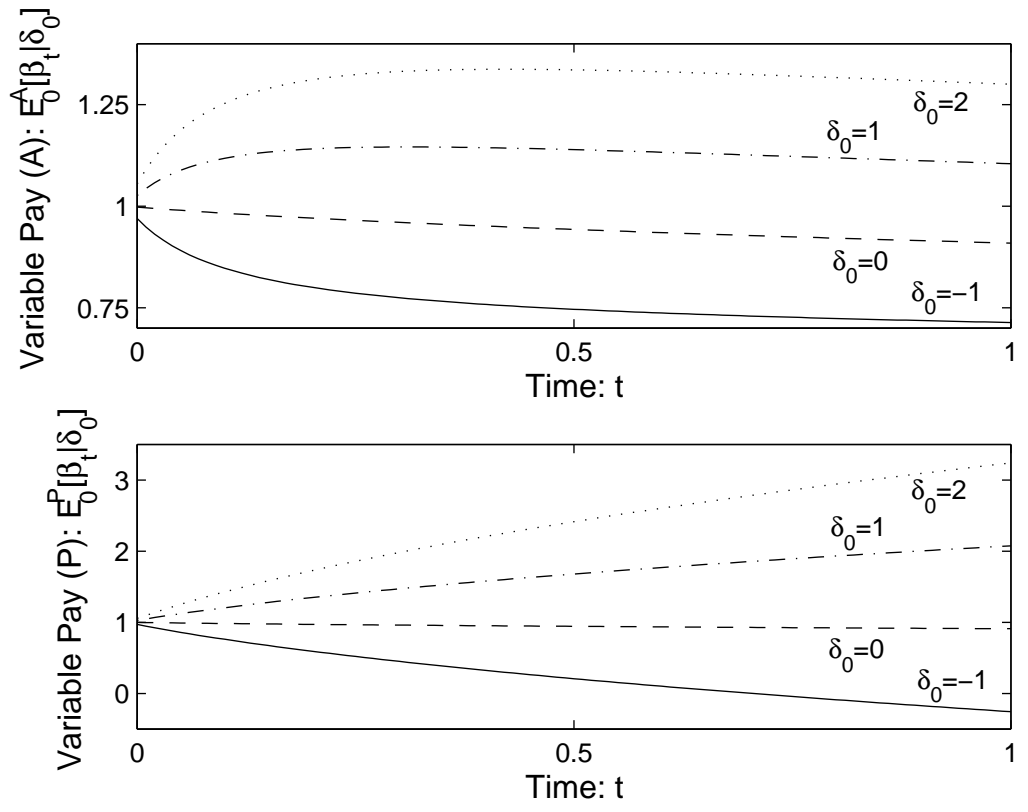

Figure 9: An impulse response function to variable pay $\left(\beta_{t}\right)$ given a shock at time 0 to beliefs. We plot both $\mathrm{E}^{\mathbb{A}}\left[\beta_{t} \mid \delta_{0}\right]$ and $\mathrm{E}^{\mathbb{P}}\left[\beta_{t} \mid \delta_{0}\right]$ for $\delta_{0}=-1,0,1,2$. We set $A=T=1, a=.1, \sigma=.8$ and $\gamma_{0}=5$. 
function more convex, as illustrated in figure 8 , and it makes the non-converge result more extreme, as illustrated in figure 9 , which can be compared to figures 4 and 5 in the secondbest. In total, the first-best has a similar dynamic structure to the second-best, but because the principal does not have to link effort and variable pay, the effects of dynamic and changing beliefs on variable pay and value are much greater.

\section{Intermediate Consumption}

In this section we will take our general model of disagreement and learning and apply it to a contracting model with intermediate consumption and effort cost. We have three purposes here: 1) demonstrate that risk aversion of the principal is not required for our results, particularly disagreement risk, 2) show that our commitment result can be generalized to allow for intermediate consumption, and 3) provide the implied technical contribution.

\subsection{The Model}

We maintain the same assumptions about the probability space, the project, and beliefs from section 2. We change only preferences, the presence of intermediate consumption and costs, and the structure of the contract.

In return for the agent's labor, the principal offers the agent a contract that specifies a terminal payment $C_{T}$, payable at time $T$, along with a flow of intermediate consumption $c$. We assume that $c$ is restricted so that $c_{t} \in \mathbb{R}$ and $c \in \mathcal{L}_{1}$. The principal is restricted to offering a contract for which $C_{T}$ is $\mathcal{Y}_{T}$-measurable and $c_{t}$ that are $\mathcal{Y}_{t}$-measurable.

The principal is risk neutral and acts to maximize

$$
\mathrm{E}^{\mathbb{P}}\left[\int_{0}^{T} e^{-r t}\left(d Y_{t}-c_{t} d t\right)-e^{-r T} C_{T}\right]
$$

while the agent acts to maximize

$$
\mathrm{E}^{\mathbb{A}}\left[\int_{0}^{T} e^{-r t}\left(u\left(c_{t}\right)-g\left(\mu_{t}\right)\right) d t+e^{-r T} U\left(C_{T}\right)\right]
$$

and $r$ is the shared rate of time discounting. 


\subsection{The Optimal Contract}

We now present the optimal dynamic contract using the utility-based "accrual" process that we used in part 3, generalize to allow for the addition of intermediate consumption:

Proposition 3 [Optimal Contracts]: Assume a given contract $\left\{C_{T}, c\right\}$ solves the principal's problem. Then the contract implements $\mu_{t}^{*}$ if and only if $C_{T}$ is the terminal value of the $C_{t}$ process with $\hat{U}=-\exp \left(-a C_{0}\right)$ and

$$
d C_{t}=-\frac{u\left(c_{t}\right)-g\left(\mu_{t}^{*}\right)}{U^{\prime}\left(C_{t}\right)} d t+\frac{r U\left(C_{t}\right)}{U^{\prime}\left(C_{t}\right)} d t+a\left(C_{t}\right) \frac{1}{2}\left(\frac{\beta_{t} \sigma_{t}}{U^{\prime}\left(C_{t}\right)}\right)^{2} d t+\frac{\beta_{t}}{U^{\prime}\left(C_{t}\right)}\left(d Y_{t}-\mu_{t}^{*} d t\right)
$$

for which $\beta\left(t, \mathcal{Y}_{t}\right)$ and $\mu_{t}^{*}$ are related $b y^{6}$

$$
\mu_{t}^{*}=j\left(\beta\left(t, \mathcal{Y}_{t}\right)\right)
$$

Furthermore,

$$
\begin{aligned}
& \mathrm{E}^{\mathbb{A}}\left[\int_{0}^{T} e^{-r t}\left(u\left(c_{t}\right)-g\left(\mu_{t}\right)\right) d t+e^{-r T} U\left(C_{T}\right) \mid \mathcal{B}_{t}, \mu_{t}=\mu_{t}^{*}\right] \\
= & \int_{0}^{t} e^{-r s}\left(u\left(c_{s}\right)-g\left(\mu_{s}^{*}\right)\right) d s+e^{-r t} U\left(C_{t}\right)
\end{aligned}
$$

As in the case of terminal consumption, when $\mu_{t}$ is drawn from a discrete or bounded set, then (23) becomes $\mu_{t}^{*}=\arg \max _{\mu_{t}} \beta\left(t, \mathcal{Y}_{t}\right) \mu_{t}-g\left(\mu_{t}\right)$ which can be substituted into (22). This will change $\mu_{t}^{*}$ from an explicit to an implicit function of $\beta_{t}$.

The form of the contract is very similar to the terminal consumption case, with two exceptions. First, because the cost of effort is now a utility cost rather than a financial cost, the agent is motivated by variable utility "pay" rather than variable cash payment. To see this, we can use an alternate formulation that takes the agent's remaining expected utility as the state variable: $\mathcal{U}_{t}=U\left(C_{t}\right)$ instead of $C_{t}$. Then

$$
d \mathcal{U}_{t}=r \mathcal{U}_{t} d t-u\left(c_{t}\right) d t+g\left(\mu_{t}^{*}\right) d t+\beta_{t}\left(d Y_{t}-\mu_{t}^{*} d t\right)
$$

and $\beta_{t}$ is the agent's utility share of the project rather than cash share.

\footnotetext{
${ }^{6}$ Recall that $j$ is the agent's inverse marginal cost of effort (3).
} 
Equation (25) also shows that the difference in beliefs now most directly affects the agent's expected level of utility. As the contract is written, the agent believes that his level of utility is a martingale; because the principal believes that part of the project output net of effort $\left(d Y_{t}-\mu_{t}^{*} d t\right)$ is predictable, the principal believes that the agent's level of expected utility declines over time when the agent is optimistic:

$$
d \mathcal{U}_{t}=r \mathcal{U}_{t} d t-u\left(c_{t}\right) d t+g\left(\mu_{t}^{*}\right) d t-\beta_{t} \sigma_{t} \delta_{t} d t+\beta_{t} \sigma_{t} d B_{t}^{\mathbb{P}}
$$

By the principal's measure, the agent overvalues variable pay by an amount $\beta_{t} \sigma_{t} \delta_{t}$, which is positive when the agent is optimistic. So, as the contract persists, the principal anticipates having to reward the agent with progressively lower levels of utility and consumption.

The second exception is that $C_{t}$ now includes the agent's intermediate consumption and effort costs. In fact, this is what allows us to generalize our commitment result. Because the potential terminal payment $C_{t}$ now includes the agent's intermediate consumption and cost of effort, it is still the case that $C_{t}$ rewards the agent for costs at the moment they are incurred. So, as shown in (24), the agent is always indifferent to quitting, being fired, or continuing to manage the project. As in the terminal consumption case, the maximization condition (23) treats the agent's maximization decision as a repeated static problem.

In the utility formulation, taking $\mathcal{U}_{t}=U\left(C_{t}\right)$ as the state variable instead of $C_{t}$, the principal will act to maximize

$$
\mathrm{E}^{\mathbb{P}}\left[\int_{0}^{T} e^{-r t}\left(\mu_{t}-c_{t}\right) d t-e^{-r T} U^{-1}\left(\mathcal{U}_{T}\right)\right]
$$

while $\mathcal{U}_{t}$ evolves as in (26).

We can now state the principal's problem analogously to the case with only terminal consumption: 
Proposition 4 [The Principal's Problem]: A contract $C_{T}$ is a solution to the principal's problem (9) if and only if

$$
\begin{array}{ll}
\beta^{*} \quad \in \quad \arg \max _{\beta, c} \mathrm{E}^{\mathbb{P}}\left[\int_{0}^{T} e^{-r t}\left(d Y_{t}-c_{t} d t\right)-e^{-r T} C_{T}\right] \\
\text { s.t. } \quad \text { (i) } d Y_{t}=\left(j\left(\beta_{t}\right)-\sigma_{t} \delta_{t}\right) d t+\sigma_{t} d B_{t}^{\mathbb{P}} \\
\quad \text { (ii) } d C_{t}=-\frac{u\left(c_{t}\right)-g\left(j\left(\beta_{t}\right)\right)}{U^{\prime}\left(C_{t}\right)} d t+\frac{r U\left(C_{t}\right)}{U^{\prime}\left(C_{t}\right)} d t+a\left(C_{t}\right) \frac{1}{2}\left(\frac{\beta_{t} \sigma_{t}}{U^{\prime}\left(C_{t}\right)}\right)^{2} d t+\frac{\beta_{t}}{U^{\prime}\left(C_{t}\right)}\left(d Y_{t}-\mu_{t}^{*} d t\right)
\end{array}
$$

where $Y_{0}=0$ and $\hat{U}=U\left(C_{0}\right)$.

The principal's problem is concave over $\mathcal{U}_{t}$.

We now proceed to examine the principal's problem with Bayesian learning.

\subsection{Discussion}

\section{Disagreement Risk}

If we make the same assumptions as in section 4 - Bayesian learning, constant $\sigma$, and quadratic cost of effort - the solution to the principal's problem is characterized by the Hamilton-Jacobi-Bellman equation

$$
\begin{aligned}
0= & \max _{\beta_{t}, c_{t}}\left[\beta_{t}-c_{t}-r F+F_{t}+F_{\mathcal{U}}\left(r \mathcal{U}_{t}-u\left(c_{t}\right)+\frac{1}{2} \beta_{t}^{2}-\beta_{t} \sigma \delta_{t}\right)\right. \\
& \left.+\frac{1}{2} F_{\mathcal{U} \mathcal{U}} \beta_{t}^{2} \sigma^{2}+\frac{1}{2} F_{\delta \delta}\left(\frac{\gamma_{t}}{\sigma}\right)^{2}+F_{\mathcal{U} \delta} \gamma_{t} \beta_{t}\right]
\end{aligned}
$$

where $e^{-r t} F\left(t, \mathcal{U}_{t}, \delta_{t}\right)$ is the value function, and $e^{-r T} F\left(T, \mathcal{U}_{T}, \delta_{T}\right)=-e^{-r T} U^{-1}\left(\mathcal{U}_{T}\right)$ is the terminal condition. Using the first order conditions, the optimal controls are given by

$$
\begin{aligned}
u^{\prime}\left(c_{t}^{*}\right) & =-\frac{1}{F_{\mathcal{U}}} \\
\beta_{t}^{*} & =\frac{1-F_{\mathcal{U}} \sigma \delta_{t}+F_{\mathcal{U} \delta} \gamma_{t}}{-F_{\mathcal{U}}-F_{\mathcal{U} \mathfrak{U}} \sigma^{2}}
\end{aligned}
$$

The key point is that disagreement risk will still exist in the economy, except that it will be driven by covariances with the agent's remaining expected utility rather than covariances with the principal's wealth level. In the previous sections, the principal's value function was 
concave over wealth. Now the principal is risk neutral, but his value function is concave over the other relevant state variable: the agent's utility. This concavity is because there is an optimal level of wealth for the agent at which the agent can still be punished with lower utility and it is not to expensive to grant the agent utility through consumption. ${ }^{7}$

Because the principal is "risk averse" with respect to the agent's wealth, he views state variables, like beliefs, as additional risk factors. The principal's certainty equivalent wealth is now made up of two factors in addition to the cost of employing the agent, as denoted by the agent's expected utility. The first factor is the principal's assessment of the project's underlying profitability. Because the principal learns, this is correlated with unexpected shocks to output. But the principal must also pay the agent for these unexpected shocks, and so the projects future profitability, according to the principal's measure, is positively correlated with the agent's remaining expected utility. The second factor is the value the principal receives from the side-bets inherent in the contract. If the agent is optimistic and there is a positive shock to the project, then the principal learns and beliefs converge. This makes side-bets less valuable at the same time the principal must increase the agent's expected utility, so the value to side-bets is negatively correlated with the agent's remaining utility.

Together, these two effects mean that the principal will still face disagreement risk even if he is risk neutral. The presence of intermediate consumption and cost of effort simply means that the principal faces this as a risk factor affecting the agent's utility and cost of employment rather than the principal's own wealth directly. As a result, the optimal level of variable pay has both "direct" and "indirect" parts. The direct term, $-F_{U} \sigma \delta_{t}$, represents the direct effect of side-bets and is positive when the agent is optimistic $\left(\delta_{t}>0\right)$. The indirect term, $F_{U \delta} \gamma_{t}$, represents the risk from the covariance of $\delta_{t}$ and $\mathcal{U}_{t}$.

\section{Welfare}

When doing welfare analysis, one must be careful in choosing the probability measure used to calculate expected utility. Throughout this paper, we are consistently agnostic with respect to whether the principal and the agent are in fact correct in their evaluation of the world. Thus, we follow Savage (1954) and Anscombe and Aumann (1963) in using the participants' subjective probabilities to evaluate their welfare.

\footnotetext{
${ }^{7}$ Please see Sannikov (2006) for a detailed explanation of how the value function behaves with homogenous beliefs and intermediate consumption.
} 
This is sensible because utility is about relative choices. In particular, we are interested in knowing the answer to this question: "what would the participants require - in terms of money - to give up the opportunity they now have?" This is a statement about how the participants value their opportunities, and so it must be taken under their own measures.

The agent obtains his reservation utility $\hat{U}$ at time 0 under his own measure. By manipulating the agent's utility evolution (25, we can see that expected continuation utility evolves according to a martingale along the optimal path:

$$
\mathrm{E}_{t}^{\mathbb{A}}\left[\int_{0}^{T} e^{-r s}\left(u\left(c_{s}\right)-g\left(\mu_{s}\right)\right) d s+e^{-r T} U\left(C_{T}\right) \mid \mathcal{B}_{t}, \mu_{t}=\mu_{t}^{*}\right]=\hat{U}+\int_{0}^{t} e^{-r s} \beta_{s} \sigma_{s} d B_{s}^{\mathbb{A}}
$$

So, while the evolution of the agent's utility function depends on the agent's beliefs, it does not directly depend on the principal's beliefs or on the difference between them. The principal takes the differences in beliefs into account in designing the contract, but the agent's welfare is pinned down by his outside option. The slope of the incentive contract $\beta_{t}$ does generally depend on the differences in beliefs, but this does not affect the growth rate of the agent's utility; it affects only its variability. In equilibrium, the agent is exactly compensated for this risk induced by steeper incentive.

In our setup, the principal effectively has all the bargaining power with respect to the heterogeneity in beliefs. This is the source of the convexity of the principal's value function: the principal is able to allocate all the gains from trade (the gains from side-bets) to himself. Employing a more or less optimistic agent can change the principal's welfare drastically, but it does not change the agent's expected welfare.

\section{Conclusion}

We have presented a model of contracting under heterogeneous beliefs in continuous time. We first imbedded a very general model of beliefs into a simple and very well understood contracting model to illustrate our results. We derive a theorem that reduces the principalagent model with belief differences to a standard dynamic programming problem. Later, we generalized the model of contracting to allow for intermediate consumption and more general utilities.

Our main result is that the principal takes advantage of disagreement in contract design, 
and differing priors can actually increase the principal's ex-ante welfare. In particular, the principal desires to sell the agent consumption in states the agent thinks are relatively more likely. In addition, the correlation between the project and beliefs can induce the principal to shift risk to or from the agent to induce changing levels of effort. There is a regime shift across moderate and large belief differences, and small shocks to beliefs can cause large variations in contract form well after beliefs have converged.

Together, our results show that dynamic considerations are important in understanding the effects of beliefs on contracts, and that those contracts can be sensitive to belief changes a well as levels. 


\section{A Proofs}

Proof of Proposition 1. Define the agent's expected utility, given that he uses $\mu=\mu^{*}$ and measured with respect to the principal's information set, as

$$
\mathcal{V}_{t} \equiv \mathrm{E}^{\mathbb{A}}\left[-\exp \left(-a\left(C_{T}-G_{T}\right)\right) \mid \mathcal{Y}_{t}, \mu=\mu^{*}\right]
$$

Since the contract is assumed to solve the principal's problem, the participation constraint must bind exactly, and so $\mathcal{V}_{0}=\hat{U}$.

Because $\mathcal{V}_{t}$ is a martingale with respect to the information set $\mathcal{Y}_{t}$ (by the law of iterated expectations), we can use a Martingale Representation Theorem (from Davis and Varaiya (1973) and updated in Revuz and Yor (2005)) to show that there exists a $\phi_{t}$ with $\phi_{t} \sigma_{t} \in \mathcal{L}_{2}$ such that

$$
d \mathcal{V}_{t}=\phi_{t}\left(d Y_{t}-\mu_{t}^{*} d t\right)
$$

where $d Y_{t}-\mu_{t}^{*} d t$ has zero drift under the agent's beliefs.

Notice that $\mathcal{V}_{T}=-\exp \left(-a\left(C_{T}-G_{T}^{*}\right)\right)$. Let us define the process $C_{t}$ so that $\mathcal{V}_{t}=$ $-\exp \left(-a\left(C_{t}-G_{t}^{*}\right)\right)$ and $\hat{U}=-\exp \left(-a C_{0}\right)$. Then $C_{T}$ must be the time $T$ value of the process $C_{t}$ (with a small abuse of notation).

Substituting $\mathcal{V}_{t}=-\exp \left(-a\left(C_{t}-G_{t}^{*}\right)\right)$ into (30) and using Ito's lemma, we find that

$$
a \exp \left(-a\left(C_{t}-G_{t}^{*}\right)\right)\left[d C_{t}-g\left(\mu_{t}^{*}\right) d t-\frac{1}{2} a\left(\operatorname{vol}\left(C_{t}\right)\right)^{2} d t\right]=\phi_{t}\left(d Y_{t}-\mu_{t}^{*} d t\right)
$$

where $\operatorname{vol}\left(C_{t}\right)$ is the volatility of $C_{t}$. Matching volatility, solving for $d C_{t}$, and substituting $\phi_{t}=\beta_{t} a \exp \left(-a\left(C_{t}-G_{t}^{*}\right)\right)$, we find

$$
d C_{t}=g\left(\mu_{t}^{*}\right) d t+a \frac{1}{2} \beta_{t}^{2} \sigma_{t}^{2} d t+\beta_{t}\left(d Y_{t}-\mu_{t}^{*} d t\right)
$$

This establishes the form of the contract. Notice that it depends on both $\mu$ (through $d Y_{t}$ ) and $\mu_{t}^{*}$.

We now use the incentive compatibility constraint to find the relationship between $\beta$ and $\mu^{*}$. This part of the proof is a standard verification theorem, and it is adapted from Vayanos and Wang (2006). 
Define the variable $\hat{\mathcal{V}}_{t}$ so that

$$
\hat{\mathcal{V}}_{t}=V\left(t, C_{t}, G_{t}\right)=-\exp \left(-a\left(C_{t}-G_{t}\right)\right)
$$

for some general $\mu$ process. Here, $C_{t}$ denotes the terminal consumption process for the control $\mu(32)$, and $\hat{\mathcal{V}}_{T}$ is the agent's final realized utility. Observe that the Hamilton-Jacobi-Bellman equation (34) can be re-written as

$$
\begin{aligned}
0=\max _{\mu} \mathrm{E}^{\mathbb{A}}\left[d \hat{\mathcal{V}}_{t} \mid \mathcal{B}_{t}\right]= & \max _{\mu_{t}}\left[V_{t}+V_{G} g\left(\mu_{t}\right)+\frac{1}{2} V_{C C}\left(\beta_{t} \sigma_{t}\right)^{2}\right. \\
& \left.+V_{C}\left(\beta_{t}\left(\mu_{t}-\mu_{t}^{*}\right)+a \frac{1}{2}\left(\beta_{t} \sigma_{t}\right)^{2}+g\left(\mu_{t}^{*}\right)\right)\right]
\end{aligned}
$$

Observe that the second-order conditions are met and that

$$
\mu_{t}^{*}=\arg \max _{\mu_{t}} \beta_{t} \mu_{t}-g\left(\mu_{t}\right)
$$

achieves the maximum $\left(\mu_{t}^{*}=j\left(\beta_{t}\right)\right)$ and sets the right-hand side of (34) equals zero. Thus, the drift of $\hat{\mathcal{V}}_{t}$ is less than or equal to zero for any $\mu_{t}$, and so

$$
\hat{\mathcal{V}}_{T} \leq \hat{\mathcal{V}}_{t}+\int_{t}^{T} \beta_{s} a \exp \left(-a\left(C_{s}-G_{s}\right)\right) \sigma_{s} d B_{s}^{\mathbb{A}}
$$

Since $\phi_{t} \sigma_{t} \in \mathcal{L}_{2}$, the above expression is integrable, and so we can take expectations:

$$
\hat{\mathcal{V}}_{t} \geq \mathrm{E}^{\mathbb{A}}\left[\hat{\mathcal{V}}_{T} \mid \mathcal{B}_{t}\right]
$$

This shows that $\hat{\mathcal{V}}_{t}$ is an upper bound on the agent's time $t$ expected utility.

Now, we repeat equations (33) and (37) for $\mu_{t}^{*}$. Since the $\mu^{*}$ solves the maximization in (34) with the right hand side equal to zero, the drift of $\hat{\mathcal{V}}_{t}$ is zero for $\mu=\mu^{*}$ and

$$
\hat{\mathcal{V}}_{t}=\mathrm{E}^{\mathbb{A}}\left[\hat{\mathcal{V}}_{T} \mid \mathcal{B}_{t}, \mu=\mu^{*}\right]
$$

This shows that the upper bound on the agent's utility is realized when $\mu=\mu^{*}$, meaning $\mu^{*}$ is the optimal control. It is unique, up to a set of measure zero, because the solution to the HJB equation was unique.

We now have two statements: First, if a contract $C_{T}$ implements $\mu_{t}^{*}$, then (32) must hold. 
Second, if (32) holds, then (35) is true if and only if $C_{T}$ implements $\mu_{t}^{*}$. Together, these imply that $C_{T}$ implements $\mu_{t}^{*}$ if and only if (32) and (35) hold.

The final equation is a re-statement of the value function and follows from the fact if $\mu=\mu^{*}$ is known, then $\mathcal{B}_{t}=\mathcal{Y}_{t}$. This completes the proof.

Proof of Proposition 2. Proposition 1 is sufficient to show that a solution to the principal's original problem (9) is also a solution to the principal's revised problem (16).

For the converse: the feasible set in the principal's revised problem (16) is (weakly) contained in the feasible set for the principal's original problem (9). Since we have shown that any optimum over the larger set (9) must be in the smaller set (16) (proposition 1) and the objective is the same, then any optimum over the smaller set must also an optimum over the larger set. This completes the proof.

Proof of Proposition 3. Define the agent's expected utility, given that he uses $\mu=\mu^{*}$ and measured with respect to the principal's information set, as

$$
\begin{aligned}
\mathcal{V}_{t} & \equiv \mathrm{E}^{\mathbb{A}}\left[\int_{0}^{T} e^{-r t}\left(u\left(c_{t}\right)-g\left(\mu_{t}\right)\right) d t+e^{-r T} U\left(C_{T}\right) \mid \mathcal{Y}_{t}, \mu_{t}=\mu_{t}^{*}\right] \\
& =\int_{0}^{t} e^{-r s}\left(u\left(c_{s}\right)-g\left(\mu_{s}\right)\right) d s+e^{-r t} \mathcal{U}_{t}
\end{aligned}
$$

$\mathcal{U}_{t}$ is the agent's remaining expected utility. Since the contract is assumed to solve the principal's problem, the participation constraint must bind exactly, and so $\mathcal{V}_{0}=\mathcal{U}_{0}=\hat{U}$.

Because $\mathcal{V}_{t}$ is a martingale with respect to the information set $\mathcal{Y}_{t}$ (by the law of iterated expectations), we can use a Martingale Representation Theorem (from Davis and Varaiya (1973) and updated in Revuz and Yor (2005)) to show that there exists a $\beta_{t}$ process with $\beta_{t} \sigma_{t} \in \mathcal{L}_{2}$ so that

$$
d \mathcal{V}_{t}=e^{-r t} \beta_{t}\left(d Y_{t}-\mu_{t}^{*} d t\right)
$$

where $d Y_{t}-\mu_{t}^{*} d t$ has zero drift under the agent's measure. Substituting (40) into (39b) and using Ito's lemma yields

$$
d \mathcal{U}_{t}=r \mathcal{U}_{t} d t-u\left(c_{t}\right) d t+g\left(\mu_{t}^{*}\right) d t+\beta_{t}\left(d Y_{t}-\mu_{t}^{*} d t\right)
$$

Notice that $\mathcal{V}_{T}=\mathcal{U}_{T}=U\left(C_{T}\right)$. Let us define the process $C_{t}$ so that $\mathcal{U}_{t}=U\left(C_{t}\right)$ and $\hat{U}=U\left(C_{0}\right)$. Then $C_{T}$ must be the time $T$ value of the process $C_{t}$ (with a small abuse of 
notation). Substituting $\mathcal{U}_{t}=U\left(C_{t}\right)$ into (41) and using Ito's lemma, find that

$$
U^{\prime}\left(C_{t}\right) d C_{t}+\frac{1}{2} U^{\prime \prime}\left(C_{t}\right)\left(\operatorname{vol}\left(C_{t}\right)\right)^{2} d t=r U\left(C_{t}\right) d t-u\left(c_{t}\right) d t+g\left(\mu_{t}^{*}\right) d t+\beta_{t}\left(d Y_{t}-\mu_{t}^{*} d t\right)
$$

where $\operatorname{vol}\left(C_{t}\right)$ is the volatility of $C_{t}$. Matching volatility, solving for $d C_{t}$, and substituting $a\left(C_{t}\right)=-\frac{U^{\prime \prime}\left(W_{t}\right)}{U^{\prime}\left(W_{t}\right)}$, we find

$$
d C_{t}=-\frac{u\left(c_{t}\right)-g\left(\mu_{t}^{*}\right)}{U^{\prime}\left(C_{t}\right)} d t+\frac{r U\left(C_{t}\right)}{U^{\prime}\left(C_{t}\right)} d t+a\left(C_{t}\right) \frac{1}{2}\left(\frac{\beta_{t} \sigma_{t}}{U^{\prime}\left(C_{t}\right)}\right)^{2} d t+\frac{\beta_{t}}{U^{\prime}\left(C_{t}\right)}\left(d Y_{t}-\mu_{t}^{*} d t\right)
$$

We now use the incentive compatibility constraint to find the relationship between $\beta$ and $\mu^{*}$. This part of the proof is a standard verification theorem, and it is adapted from Vayanos and Wang (2006).

Define the variable $\hat{\mathcal{V}}_{t}$ so that

$$
\hat{\mathcal{V}}_{t}=\int_{0}^{t} e^{-r s}\left(u\left(c_{s}\right)-g\left(\mu_{s}\right)\right) d s+e^{-r t} \mathcal{U}_{t}
$$

for some general $\mu$ process. Here, $\mathcal{U}_{t}$ denotes the terminal utility process for the control $\mu$ (41). Observe that the Hamilton-Jacobi-Bellman equation can be written as

$$
0=\max _{\mu} \mathrm{E}^{\mathbb{A}}\left[d \hat{\mathcal{V}}_{t} \mid \mathcal{B}_{t}\right]=\max _{\mu_{t}} e^{-r t}\left[-g\left(\mu_{t}\right)+g\left(\mu_{t}^{*}\right)+\beta_{t}\left(\mu_{t}-\mu_{t}^{*}\right)\right]
$$

Observe that the second-order conditions are met and that

$$
\mu_{t}^{*}=\arg \max _{\mu_{t}} \beta_{t} \mu_{t}-g\left(\mu_{t}\right)
$$

achieves the maximum $\left(\mu_{t}^{*}=j\left(\beta_{t}\right)\right)$ and sets the right-hand side of (45) equals zero. Thus, the drift of $\hat{\mathcal{V}}_{t}$ is less than or equal to zero for any $\mu_{t}$, and so

$$
\hat{\mathcal{V}}_{T} \leq \hat{\mathcal{V}}_{t}+\int_{t}^{T} \beta_{s} \sigma_{s} d B_{s}^{\mathbb{A}}
$$

Since $\beta_{t} \sigma_{t} \in \mathcal{L}_{2}$, the above expression is integrable, and so we can take expectations:

$$
\hat{\mathcal{V}}_{t} \geq \mathrm{E}^{\mathbb{A}}\left[\hat{\mathcal{V}}_{T} \mid \mathcal{B}_{t}\right]
$$

This shows that $\hat{\mathcal{V}}_{t}$ is an upper bound on the agent's time $t$ expected utility. 
Now, we repeat equations (44) and (48) for $\mu_{t}^{*}$. Since the $\mu^{*}$ solves the maximization in (45) with the right hand side equal to zero, the drift of $\hat{\mathcal{V}}_{t}$ is zero for $\mu=\mu^{*}$ and

$$
\hat{\mathcal{V}}_{t}=\mathrm{E}^{\mathbb{A}}\left[\hat{\mathcal{V}}_{T} \mid \mathcal{B}_{t}, \mu=\mu^{*}\right]
$$

This shows that the upper bound on the agent's utility is realized when $\mu=\mu^{*}$, meaning $\mu^{*}$ is the optimal control. It is unique, up to a set of measure zero, because the solution to the HJB equation was unique.

We now have two statements: First, if a contract $\left\{C_{T}, c\right\}$ implements $\mu_{t}^{*}$, then (43) must hold. Second, if (43) holds, then (46) is true if and only if $\left\{C_{T}, c\right\}$ implements $\mu_{t}^{*}$. Together, these imply that $\left\{C_{T}, c\right\}$ implements $\mu_{t}^{*}$ if and only if (43) and (46) hold.

The final equation is a re-statement of the value function and follows from the fact if $\mu=\mu^{*}$ is known, then $\mathcal{B}_{t}=\mathcal{Y}_{t}$. This completes the proof.

Proof of Proposition 4. This proof follows directly from the arguments in the proof of proposition 2. For the added concavity result, use the alternate formulation involving $\mathcal{U}$, observe that the principal is taking the maximum over a concave integrand $-u(\cdot),-g(\cdot)$, and $-U^{-1}(\cdot)$ are all concave - and apply the textbook concavity agrement. 


\section{References}

Acemoglu, Daron, Victor Chernozhukov, and Muhamet Yildiz, 2007, Learning and Disagreement in an Uncertain World, Working Paper, MIT.

Anscombe, Frank, and Robert Aumann, 1963, A Definition of Subjective Probability, Annals of Mathematical Statistics 34, 199-205.

Aumann, Robert, 1976, Agreeing to Disagree, Annals of Statistics 4, 1236-1239.

Baker, Malcolm, Richard Ruback, and Jeffrey Wurgler, 2006, Behavioral Corporate Finance: A Survey, in Espen Eckbo, eds.: The Handbook of Corporate Finance (Elsevier/North Holland, ).

Barberis, Nicholas, and Richard Thaler, 2003, A Survey of Behavioral Finance, in G.M. Constantinides, M. Harris, and R. Stultz, eds.: Handbook of the Economics of Finance (Elsevier Science B.V., Amsterdam ).

Ben-David, Itzhak, John R. Graham, and Campbell R. Harvey, 2006, Managerial Overconfidence and Coporate Policies, Working Paper.

Benabou, Roland, and Jean Tirole, 2002, Self-Confidence and Personal Motivation, Quarterly Journal of Economics 117, 871-915.

Bolton, Patrick, Jose Scheinkman, and Wei Xiong, 2006, Executive Compensation and ShortTermist Behavior in Speculative Markets, Review of Financial Studies 73, 577-610.

Cvitanić, Jakša, and Jianfeng Zhang, 2006, Optimal Compensation with Adverse Selection and Dynamic Actions, Working Paper, SSRN.

Davis, Mark H., and Pravin Varaiya, 1973, Dynamic Programming Conditions for Partially Observable Stochastic Systems, SIAM Journal on Control 11, 226-261.

Gervais, Simon, and Itay Goldstein, 2004, Overconfidence and Team Coordination, Working Paper.

Gervais, Simon, John Heaton, and Terrance Odean, 2006, Overconfidence, Investment Policy, and Manager Welfare, Working Paper, Duke University.

Gul, Faruk, 1998, A Comment on Aumann's Baysian View, Econometrica 66, 923-927. 
Holmstrom, Bengt, and Paul Milgrom, 1987, Aggregation and Linearity in the Provision of Intertemporal Incentives, Econometrica 55, 303-328.

Ito, Takatoshi, 1990, Foreign Exchange Rate Expectations: Micro Survey Data, American Economic Review 80, 434-449.

Jenter, Dirk, 2004, Market Timing and Managerial Portfolio Decisions, Journal of Finance 60, 1903-1947.

Landier, Augustin, and David Thesmar, 2006, Contracting with Optimistic Entrepreneurs: Theory and Evidence, Review of Financial Studies forthcoming.

Liptser, Robert, and Albert Shiryaev, 2000, Statistics of Random Processes. (Springer-Verlag Berlin) second edn.

Milgrom, Paul, and Nancy Stokey, 1982, Information, Trade and Common Knowledge, Journal of Economic Theory 26, 17-27.

Mullainathan, Sendhil, and Richard Thaler, 2001, Behavioral Economics, in International Encyclopedia of Social Sciences (Pergamon Press, ).

Odean, Terrance, 1999, Do Investors Trade Too Much?, American Economic Review 89, $1279-1298$.

Revuz, Daniel, and Marc Yor, 2005, Continuous Martingales and Brownian Motion vol. 293 of A Series of Comprehensive Studies in Mathematics. (Springer-Verlag Berlin) third edn.

Sannikov, Yuliy, 2006, A Continuous Time Version of the Principal-Agent Problem, Working Paper, Stanford University.

Savage, Leonard, 1954, The Foundations of Statistics. (Wiley New York).

Schättler, Heinz, and Jaeyoung Sung, 1993, The First Order Approach to the ContinuousTime Principal-Agent Problem with Exponential Utility, Journal of Economic Theory 61, $331-371$.

Van den Steen, Eric, 2001, Essays on the Managerial Implications of Differing Priors, Ph.D. Dissertation, Stanford University. 
Vayanos, Dimitri, and Jiang Wang, 2006, Capital Market Theory, Manuscript in preparation, LSE and MIT.

Westerfield, Mark, 2006, Dynamic Contracts with Hidden Actions in Continuous Time, Working Paper, SSRN.

Yildiz, Muhamet, 2003, Bargaining Without a Common Prior: An Immediate Agreement Theorem, Econometrica 71, 793-811. 